\title{
Alpha-particle velocity-space diagnostic in ITER
}

Salewski, M.; Nocente, M.; Madsen, B.; Abramovic, I.; Fitzgerald, M.; Gorini, G.; Hansen, P.C.; Heidbrink, W.W.; Jacobsen, A.S.; Jensen, T.

Total number of authors:

26

Published in:

Nuclear Fusion

Link to article, DOI:

10.1088/1741-4326/aace05

Publication date:

2018

Document Version

Peer reviewed version

Link back to DTU Orbit

Citation (APA):

Salewski, M., Nocente, M., Madsen, B., Abramovic, I., Fitzgerald, M., Gorini, G., Hansen, P. C., Heidbrink, W. W., Jacobsen, A. S., Jensen, T., Kiptily, V. G., Klinkby, E. B., Korsholm, S. B., Kurki-Suonio, T., Larsen, A. W., Leipold, F., Moseev, D., Nielsen, S. K., Pinches, S. D., ... Tardocchi, M. (2018). Alpha-particle velocity-space diagnostic in ITER. Nuclear Fusion, 58(9), [096019]. https://doi.org/10.1088/1741-4326/aace05

\section{General rights}

Copyright and moral rights for the publications made accessible in the public portal are retained by the authors and/or other copyright owners and it is a condition of accessing publications that users recognise and abide by the legal requirements associated with these rights.

- Users may download and print one copy of any publication from the public portal for the purpose of private study or research.

- You may not further distribute the material or use it for any profit-making activity or commercial gain

- You may freely distribute the URL identifying the publication in the public portal 
16 May 2018

\title{
Alpha-particle velocity-space diagnostic in ITER
}

\author{
M Salewski ${ }^{1}$, M Nocente ${ }^{2,3}$, B Madsen ${ }^{1}$, I Abramovic ${ }^{4}$, \\ M Fitzgerald ${ }^{5}$, G Gorini ${ }^{2,3}$, PC Hansen ${ }^{6}$, WW Heidbrink ${ }^{7}$, \\ AS Jacobsen ${ }^{8}$, T Jensen ${ }^{1}$, VG Kiptily ${ }^{5}$, EB Klinkby ${ }^{9}$, \\ SB Korsholm ${ }^{1}$, T Kurki-Suonio ${ }^{10}$, AW Larsen ${ }^{1}$, F Leipold ${ }^{1}$, \\ D Moseev ${ }^{4}$, SK Nielsen ${ }^{1}$, SD Pinches ${ }^{11}$, J Rasmussen ${ }^{1}$, \\ M Rebai ${ }^{2,3}$, M Schneider ${ }^{11}$, A Shevelev ${ }^{12}$, S Sipilä ${ }^{10}$, M Stejner ${ }^{1}$, \\ M Tardocchi ${ }^{3}$
}

${ }^{1}$ Department of Physics, Technical University of Denmark, Kgs. Lyngby, Denmark

${ }^{2}$ Department of Physics, University of Milano Bicocca, Milano, Italy

${ }^{3}$ Istituto di Fisica del Plasma, Consiglio Nazionale delle Ricerche, Milano, Italy

${ }^{4}$ Max-Planck-Institut für Plasmaphysik, Greifswald, Germany

${ }^{5}$ CCFE, Culham Science Centre, Abingdon, Oxon, UK

${ }^{6}$ Department of Applied Mathematics and Computer Science, Technical University of Denmark, Kgs. Lyngby, Denmark

7 Department of Physics and Astronomy, University of California Irvine, Irvine, CA, USA

8 Max-Planck-Institut für Plasmaphysik, Garching, Germany

${ }^{9}$ Center for Nuclear Technologies, Technical University of Denmark, Roskilde, Denmark

${ }^{10}$ Department of Applied Physics, Aalto University, Aalto, Finland

11 ITER Organization, Route de Vinon-sur-Verdon, CS 90 046, 13067 St. Paul-lezDurance Cedex, France

12 Ioffe Institute, Polytekhnicheskaya 26, St Petersburg, 194021, Russia

E-mail: msal@fysik.dtu.dk

\begin{abstract}
We discuss $\alpha$-particle velocity-space diagnostic in ITER based on the planned collective Thomson scattering (CTS) and $\gamma$-ray spectrometry (GRS) systems as well as ASCOT simulations of the $\alpha$-particle distribution function. GRS is sensitive to $\alpha$-particles with energies $E \gtrsim 1.7 \mathrm{MeV}$ at all pitches $p$, and CTS for $E \gtrsim 0.3 \mathrm{MeV}$ and $|p| \lesssim 0.9$. The remaining velocity space is not observed. GRS and CTS view the plasma (almost) perpendicularly to the magnetic field. Hence we cannot determine the sign of the pitch of the $\alpha$-particles and cannot distinguish co- and counter-going $\alpha$ particles with the currently planned $\alpha$-particle diagnostics. Therefore we can only infer the sign-insensitive $2 \mathrm{D}$ distribution function $f(E,|p|)$ by velocity-space tomography for $E \gtrsim 1.7 \mathrm{MeV}$. This is a serious limitation, since co- and counter-going $\alpha$-particle populations are expected to have different birth rates and neoclassical transport as well as different anomalous transport due to interaction with modes such as Alfvén eigenmodes. We propose the installation of an oblique GRS system on ITER to allow us to diagnostically track such anisotropy effects and to infer the full, sign-sensitive $f(E, p)$ for $E \gtrsim 1.7 \mathrm{MeV}$. $\alpha$-particles with $E \lesssim 1.7 \mathrm{MeV}$ are diagnosed by CTS only,
\end{abstract}


which does not allow velocity-space tomography on its own. Nevertheless, we show that measurements of the $\alpha$-particle energy spectrum, which is an ITER measurement requirement, are now feasible for $E \gtrsim 0.3 \mathrm{MeV}$ using a velocity-space tomography formalism assuming isotropy in velocity space.

\section{Introduction}

A new era of burning plasmas is approaching with the planned DT experiments at JET $[1,2]$ and the construction of the ITER tokamak [3]. The ultimate goal for ITER is a fusion power of $P_{\text {fus }}=500 \mathrm{MW}$ for an auxiliary heating power of $P_{\text {aux }}=50 \mathrm{MW}$ which gives a power amplification of $Q=P_{\text {fus }} / P_{a u x}=10$ [4]. Many present designs for the next-step device DEMO hope for $Q=20-50$ [5-7]. Such plasmas are primarily heated by $\alpha$-particles generated in the fusion reaction $\mathrm{D}(\mathrm{T}, \mathrm{n}) \alpha$. Since the fusion born $\alpha$-particle power is $P_{\alpha}=P_{\text {fus }} / 5$, the plasma self-heating fraction $\eta_{\alpha}$ for well-confined energetic particles is

$$
\eta_{\alpha}=\frac{P_{\alpha}}{P_{\alpha}+P_{a u x}}=\frac{Q}{Q+5} .
$$

The plasma self-heating fraction is an alternative way to state the goal to achieve $Q=10$ which highlights the prominent role of $\alpha$-particles for the plasma heating [4]. The importance of plasma self-heating by $\alpha$-particles increases with $Q$ (figure 1 ). The fusion power world record discharge at JET in $1997 \mathrm{had} \eta_{\alpha}=11 \%(Q=0.64)$ just below 'break-even' at $\eta_{\alpha}=17 \%(Q=1)$ [8]. Burning plasmas are predominantly self-heated by $\alpha$-particles $\left(\eta_{\alpha}>50 \%, Q>5\right)$, as in ITER $\left(\eta_{\alpha}=67 \%\right)$ or DEMO $\left(\eta_{\alpha}=80-91 \%\right)$. At 'ignition' the plasma is completely self-heated $\left(\eta_{\alpha}=100 \%\right.$, $Q \rightarrow \infty)$. Burning plasmas will offer new challenges since the temperature and density profiles are self-consistently determined by $\alpha$-particle heating rather than controlled by auxiliary heating. Furthermore, MeV-range ions may drive a zoo of instabilities deteriorating the plasma performance [9-19]. Due to the central role of $\alpha$-particles to achieve $Q=10$ and the possible anomalous transport due to instabilities, the diagnostic of $\alpha$-particles in ITER will be essential.

In the most basic modelling, $\alpha$-particles are assumed to follow a classical, isotropic slowing-down distribution. However, neoclassical transport theory and the anisotropic $\alpha$-particle birth profiles in ITER suggest that the $\alpha$-particle distribution will be anisotropic [20]. Furthermore, the basic physics of wave-particle interaction suggests that particles close to resonance conditions interact most strongly with modes. These resonance conditions are different for co- and counter-going $\alpha$-particles such that the expected anomalous wave-induced transport is thought to be substantially different for co- and counter-going particles. However, these two groups of particles cannot be told apart with the currently planned set of $\alpha$-particle diagnostics. In this paper we hence propose the installation of an extra fast-ion diagnostic at ITER which would allow us to tell co- and counter-going $\alpha$-particles apart. 


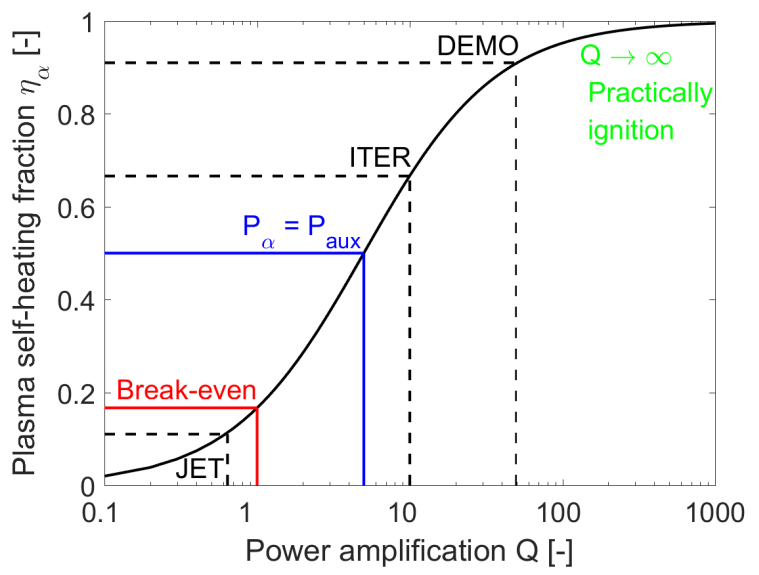

Figure 1. Plasma self-heating fraction $\eta_{\alpha}$ as function of the power amplification $Q$. Plasmas are predominantly heated by MeV-range $\alpha$-particles for $Q>5$.

Confined $\alpha$-particles in ITER are expected to be diagnosed by $\gamma$-ray spectrometry (GRS) [21-24] and collective Thomson scattering (CTS) [25-30]. In GRS measurements, the $\gamma$-rays emitted by fusion plasmas are spectrally analyzed $[31,32]$. The largest $\gamma$-ray fluxes today are achieved at JET. Early GRS measurements at JET had a moderate spectral resolution [33-41]. Developments in detectors now allow a much higher spectral resolution such that the Doppler shapes can be analyzed [22,42-51]. The high nuclear reaction rates in the upcoming DT campaign at JET $[1,2]$ and later in burning plasmas at ITER and DEMO will further enhance the $\gamma$-ray emission [21,52].

In CTS measurements, scattered electromagnetic waves are spectrally analyzed. Fast-ion CTS measurements were first done at JET [53], and nowadays on ASDEX Upgrade [54-57] and LHD [58]. Wendelstein 7-X is also equipped with CTS [59,60]. In this paper we demonstrate various ways to determine $\alpha$-particle velocity distribution functions by integrated data analysis of the GRS and CTS spectra based on velocityspace tomography $[57,61-76]$. The velocity-space tomography technique allows the measurement of $\alpha$-particle densities and energy spectra, which are ITER measurement requirements [77].

Section 2 gives a brief overview of the planned GRS and CTS systems at ITER. In section 3 we illustrate their velocity-space sensitivities. Section 4 presents expected results of velocity-space tomography at ITER and demonstrates that the sign of the pitch cannot be determined, unless an extra fast-ion diagnostic with an oblique view is added. The pitch is defined as $p=v_{\|} / v$ where $v_{\|}$is the velocity component along the magnetic field and $v$ the velocity magnitude. This limitation is explained in section 5 . In section 6 we compute reconstructions of $\alpha$-particle distribution functions simulated by ASCOT for the baseline and the hybrid scenario which do show significant asymmetry in pitch. Section 7 discusses further sources of such anisotropy in the $\alpha$-particle distribution and highlights the importance of measurements that are sensitive to the sign of the pitch. Section 8 presents a way to measure $\alpha$-particle energy spectra based on CTS or GRS 
measurements in one view by assuming isotropy in velocity space as prior information. Conclusions are drawn in section 9.

\section{Diagnostics for confined $\alpha$-particles at ITER}

For 2D velocity-space or 3D phase-space studies, spectra are of particular interest $[62,78]$. The currently planned GRS and CTS systems for $\alpha$-particle diagnostic are illustrated in figure 2. The CTS system at ITER has several measurement volumes distributed along the $60 \mathrm{GHz}, 1 \mathrm{MW}$ probe beam where it overlaps with the fields of view of highly sensitive radiometers [25-30]. We use the central measurement volume illustrated in figure 2. CTS diagnostics measure spectra of scattered radiation which are sensitive to the projection of the fast-ion velocity distribution function in the measurement volume along $\mathbf{k}^{\delta}=\mathbf{k}^{s}-\mathbf{k}^{i}$. $\mathbf{k}^{s}$ and $\mathbf{k}^{i}$ are the wave vectors of the scattered and incident waves, respectively. In CTS the probing radiation is Doppler-shifted according to $\nu^{\delta}=u\left|\mathbf{k}^{\delta}\right| / 2 \pi$ where $u$ is the projected velocity of the fast ion onto $\mathbf{k}^{\delta}$. The angle

$\phi$ between the magnetic field and $\mathbf{k}^{\delta}$ determines together with the observed Doppler shift the interrogation region of the diagnostic in velocity space (see section 3 ). The central measurement volume of the ITER CTS system has $\phi=97^{\circ}$.

The GRS system has just completed its conceptual design phase and is planned to consist of up to two perpendicular detector arrays, one vertical and one radial, out of which we select the views going through the plasma core. The lines-of-sight of both systems are about perpendicular to the magnetic field in the plasma core. GRS measurements are in principle sensitive along the entire lines-of-sight of the detectors, but the $\gamma$-ray production is strongly biased towards the plasma center where most fusion reactions occur. $\alpha$-particle measurements at ITER are mainly foreseen by GRS measurements of the two-step reaction ${ }^{9} \mathrm{Be}(\alpha, \mathrm{n} \gamma){ }^{12} \mathrm{C}$ which produces $\gamma$-rays at $4.44 \mathrm{MeV}$ and $3.2 \mathrm{MeV}$ [79]. The $4.44 \mathrm{MeV} \gamma$-rays originate from the decay of ${ }^{12} \mathrm{C}$ from the first excited state, and the $3.2 \mathrm{MeV}$ from the decay from the second to the first excited state. The second excited state is expected to get populated mostly due to $\alpha$-particles with energies larger than $3.4 \mathrm{MeV}$. Ideally, high-resolution GRS measurements resolve the spectral shapes of these $\gamma$-ray peaks which are sensitive to the velocities of the $\alpha$-particles. In this paper we do not consider the experimental challenges that must be overcome $[24,45,80,81]$, but we assume that both GRS and CTS deliver optimal measurements according to design.

\section{Velocity-space coverage of $\alpha$-particle diagnostics at ITER}

The diagnostic velocity-space coverage can be analyzed using weight functions which have been studied for the major confined fast-ion diagnostics [61,64,82-86], recently also in 3D phase-space [78]. A few illustrative examples for GRS and CTS weight functions at ITER $[24,61]$ are presented in figures 3 and 4 . We expect detectable signals for the Doppler shifts chosen in figures 3 and 4 . The amplitude in the colored regions 


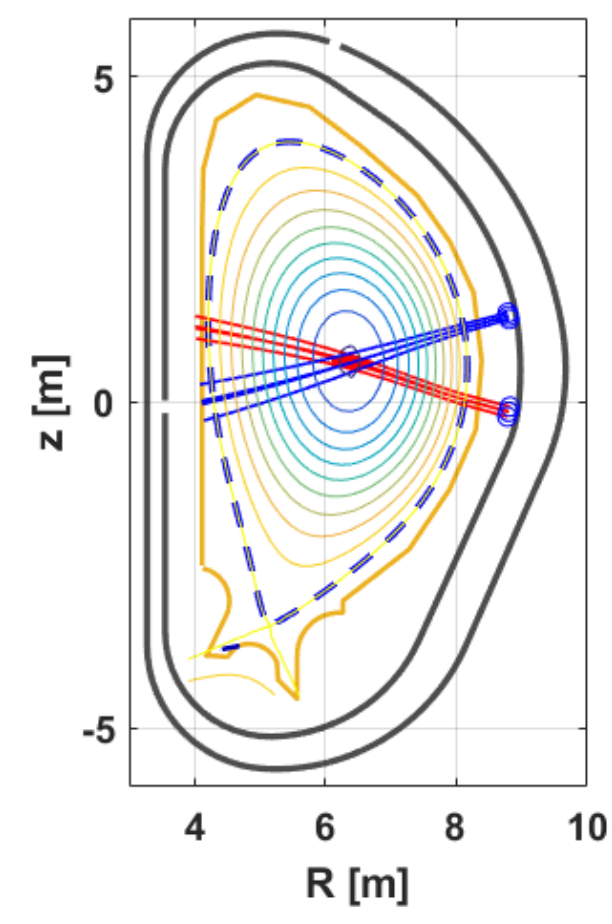

(a) CTS

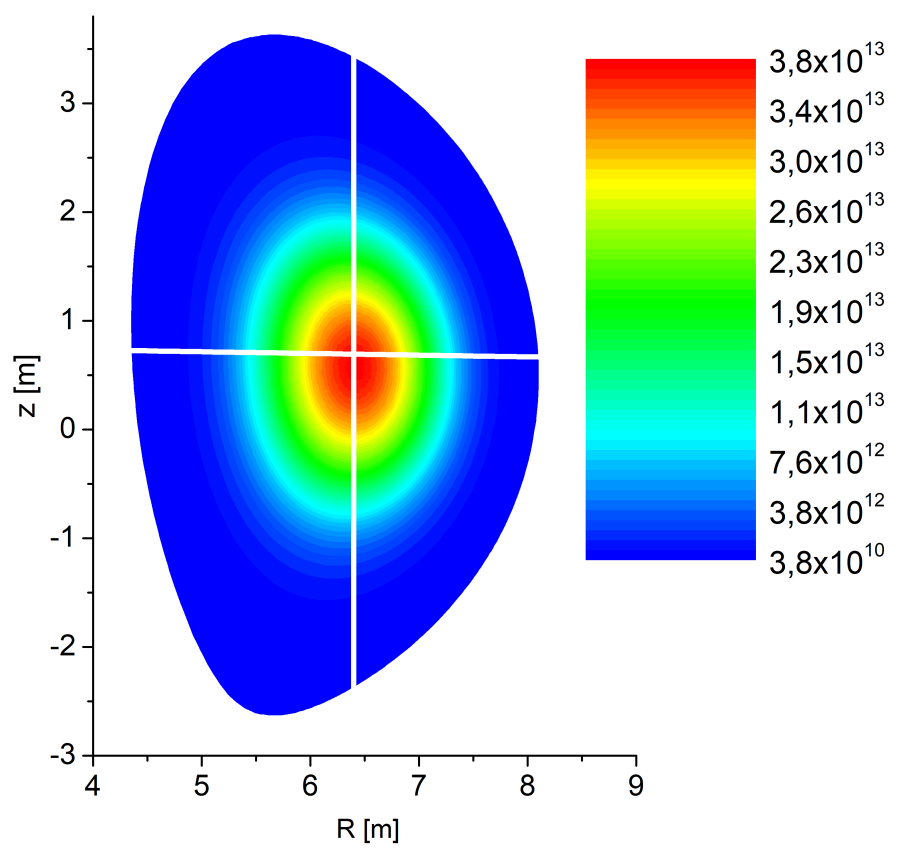

(b) GRS

Figure 2. Geometries of the $\alpha$-particle diagnostic systems planned for ITER in the baseline scenario. (a) Central measurement volume of the CTS system. The probe beam is illustrated in blue, and the central receiver beam is illustrated in red. The measurement volume is located at the intersection of the probe and receiver beams. Magnetic flux surfaces are indicated. (b) Vertical and radial GRS lines-of-sight going through the plasma center. The colours indicate the predicted $\gamma$-ray emissivity $\left[\gamma^{\prime} \mathrm{s} / \mathrm{m}^{3} \mathrm{~s}\right]$.

shows the detectable signal per ion and is thus a measure of the velocity-space sensitivity of the diagnostic. The white regions are not observed.

GRS weight functions for ITER are up-down symmetric about $p=0$ (figure 3). As GRS relies on the Doppler shift, it is impossible to tell in perpendicular views, if a given particle traverses the detector along or against the direction of the magnetic field. Therefore an ion with a given pitch $p_{1}$ generates the same signal as an ion with pitch $-p_{1}$ which means that the perpendicular GRS at ITER cannot tell co- and counter-going ions apart. Otherwise, the GRS weight functions actually have considerable selectivity in pitch. At the nominal $\gamma$-ray peak energy, the sensitivity of co- and counter-going ions is largest whereas at large Doppler shifts the sensitivity to trapped ions is largest. This pitch selectivity of the two-step reaction GRS weight functions ultimately originates from the conservation of energy and momentum [84].

Figure 3(a)-(c) shows typical GRS weight functions for the $\gamma$-ray peak at $3.2 \mathrm{MeV}$ which is most sensitive to $\alpha$-particles at energies above about $3.4 \mathrm{MeV}$ which is just below the $\alpha$-particle birth energy. The sensitivity decreases rapidly for lower $\alpha$-particle energies. $\alpha$-particles with energies larger than their birth energy at $3.5 \mathrm{MeV}$, e.g. due 
to wave heating in the ion cyclotron range of frequencies (ICRF), the Gaussian form of the birth energy distribution or anomalous effects, could easily be detected with this reaction. It will therefore be effective in reconstructions of the velocity-space above the nominal $\alpha$-particle birth energy.

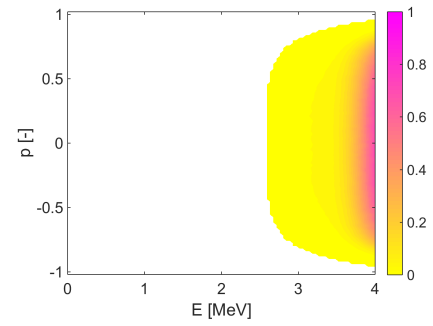

(a) $\Delta E_{\gamma}= \pm 20 \mathrm{keV}$

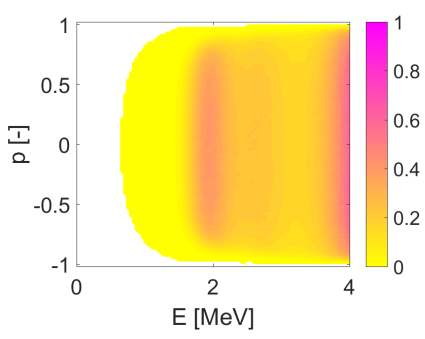

(d) $\Delta E_{\gamma}= \pm 50 \mathrm{keV}$

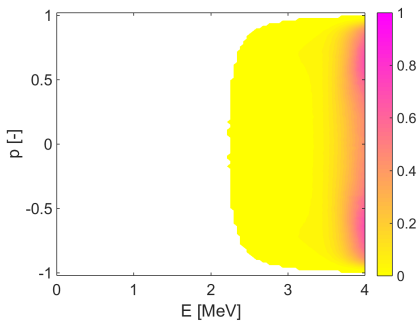

(b) $\Delta E_{\gamma}= \pm 10 \mathrm{keV}$

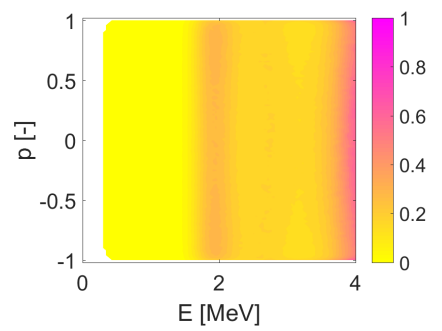

(e) $\Delta E_{\gamma}= \pm 30 \mathrm{keV}$

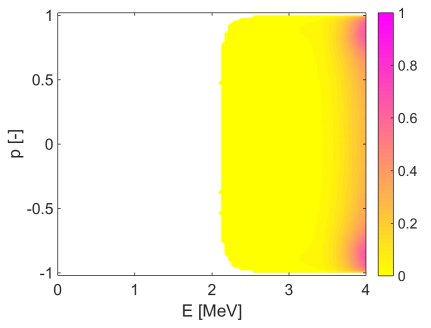

(c) $\Delta E_{\gamma}=0 \mathrm{keV}$

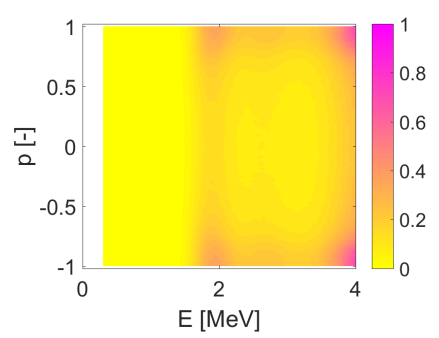

(f) $\Delta E_{\gamma}=0 \mathrm{keV}$

Figure 3. GRS weight functions [a.u.] for $\phi=90^{\circ}$ and different Doppler shifts $\Delta E_{\gamma}$ for the $\gamma$-ray peaks at $3.2 \mathrm{MeV}$ (a-c) and at $4.44 \mathrm{MeV}(\mathrm{d}-\mathrm{f}) .(E, p)$ are the energy and the pitch of the $\alpha$-particles. The pitch sensitivities change substantially from the wings to the center of the relevant spectral line. All weight functions are up-down symmetric about $p=0$. The weight functions for positive Doppler shifts are identical to those for the corresponding negative Doppler shifts.

Weight functions for the $4.44 \mathrm{MeV} \gamma$-ray peak (figure 3(d)-(f)) have some similarities with those for the $3.2 \mathrm{MeV}$ peak. However, there are important differences due to the different reaction cross sections of the two reactions. Nuclear resonances boost the sensitivity of the $4.44 \mathrm{MeV} \gamma$-ray peak near $\alpha$-particle energies of $2 \mathrm{MeV}$ and $4 \mathrm{MeV}$. The sensitivity decreases rapidly for energies below $2 \mathrm{MeV}$ such that this reaction is sensitive down to about $1.7 \mathrm{MeV}$. The sensitivity in the region between the $2 \mathrm{MeV}$ and $4 \mathrm{MeV}$ resonances should be sufficient for velocity-space tomography.

The viewing angle of CTS is $\phi=97^{\circ}$, and hence the ITER CTS system can practically be regarded as a perpendicular system. The difference from a truly perpendicular view is reflected in the slight asymmetry of the CTS weight functions about $p=0$ (figure 4). The weight functions are slightly lopsided towards negative pitches for positive projected velocities $u$ and towards positive pitches for negative $u$. This gives us the theoretical possibility to detect asymmetry in the velocity distribution function about $p=0$. However, the asymmetry in the weight functions is small, and considering realistic noise levels it turns out to be difficult to determine the sign of the pitch of a measured ion. 


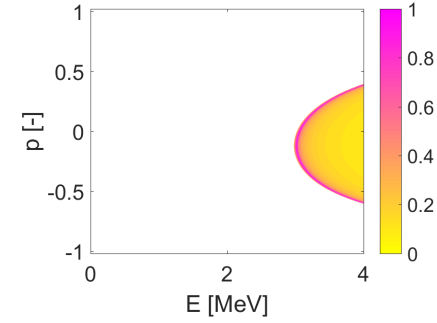

(a) $u=12 \times 10^{6} \mathrm{~m} / \mathrm{s}$

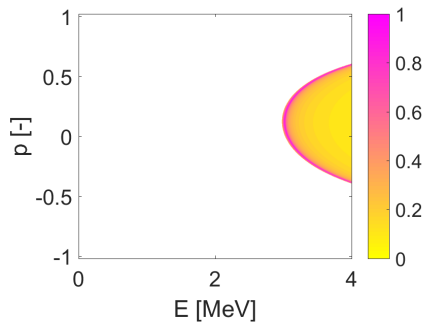

(d) $u=-12 \times 10^{6} \mathrm{~m} / \mathrm{s}$

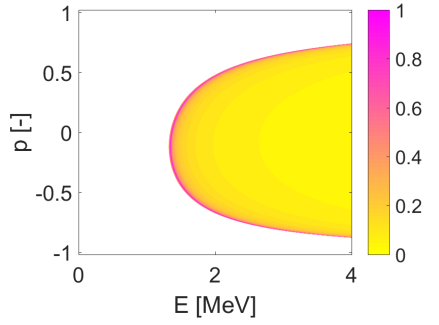

(b) $u=8 \times 10^{6} \mathrm{~m} / \mathrm{s}$

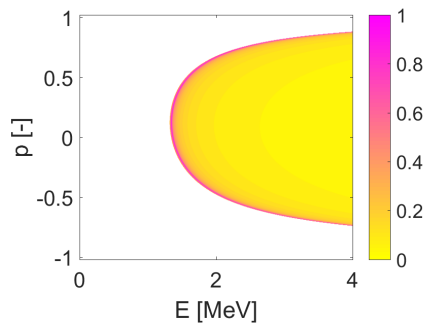

(e) $u=-8 \times 10^{6} \mathrm{~m} / \mathrm{s}$

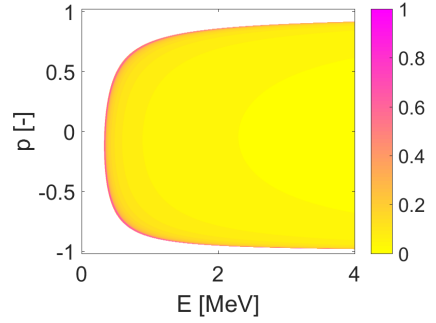

(c) $u=4 \times 10^{6} \mathrm{~m} / \mathrm{s}$

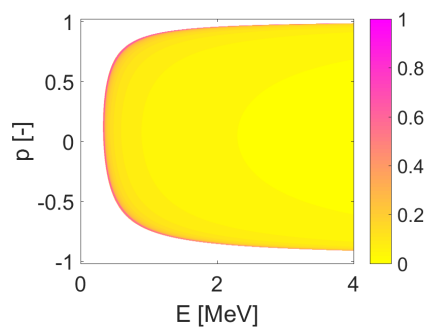

(f) $u=-4 \times 10^{6} \mathrm{~m} / \mathrm{s}$

Figure 4. CTS weight functions [a.u.] for $\phi=97^{\circ}$ and different projected velocities $u$. $(E, p)$ are the energy and the pitch of the $\alpha$-particles. Due to the almost perpendicular observation angle, the weight functions for positive and negative $u$ are very similar but not identical.

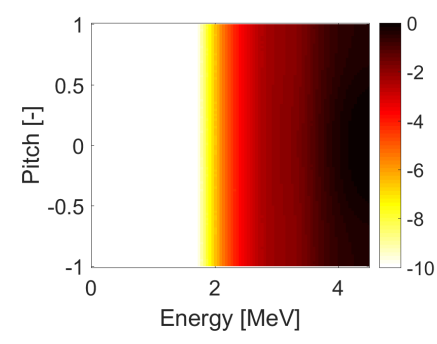

(a)

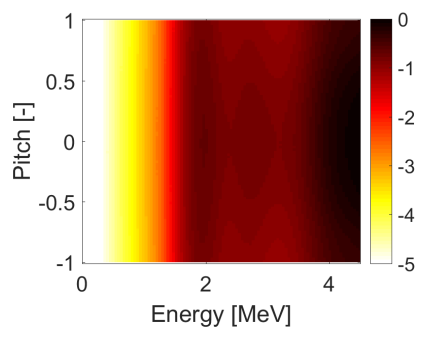

(b)

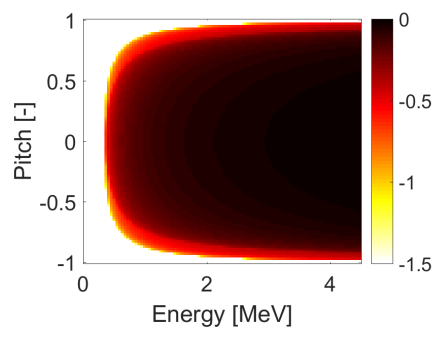

(c)

Figure 5. Illustration of gross velocity-space sensitivity of individual fast-ion diagnostics at ITER. $(E, p)$ are the energy and the pitch of the $\alpha$-particles. a) GRS at 3.2 MeV. b) GRS at $4.44 \mathrm{MeV}$. c) CTS. The sensitivity functions are normalized. The color scale shows the base ten logarithm.

A measure of the gross velocity-space sensitivity is obtained by computing weight functions covering the entire accessible spectral range. This is equivalent to summing the weight functions associated with each experimentally accessible data point in the spectrum. The weight functions are normalized by the noise levels of their associated measurements before computing the sum which is here assumed to be $10 \%$ [63]. Figure 5 illustrates such gross sensitivities for the three spectra (out of which each GRS spectrum will be measured by two detectors simultaneously).

Basic features of the individual weight functions enter the gross velocity-space sensitivity of the $\gamma$-ray peaks at 3.2 MeV and 4.44 MeV (figures 5(a) and (b)). The gross velocity-space sensitivities largely resemble what is expected from the energy dependence 


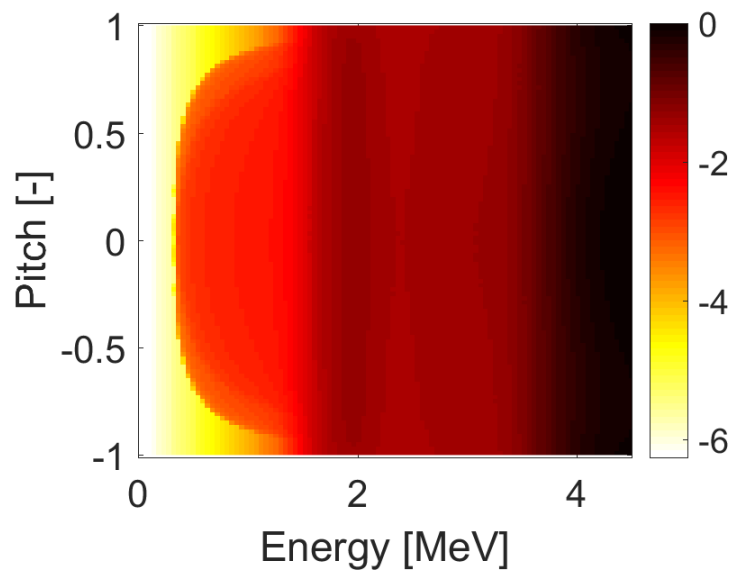

Figure 6. Illustration of the gross velocity-space sensitivity of CTS and GRS together. $(E, p)$ are the energy and the pitch of the $\alpha$-particles. The sensitivity functions are normalized. The color scale shows the base ten logarithm.

of the reaction cross sections. The $3.2 \mathrm{MeV}$ peak has little sensitivity at energies below $\sim 3.4 \mathrm{MeV}$ and becomes very sensitive above the $\alpha$-particle birth energy. The $4.44 \mathrm{MeV}$ peak is strongly sensitive at the resonances at $2 \mathrm{MeV}$ and $4 \mathrm{MeV}$ and has a good sensitivity between the resonances.

The CTS diagnostic (figure 5(c)) can detect $\alpha$-particles despite the thermal deuterium down to about $0.3 \mathrm{MeV}$ for $p \sim 0$. This energy is based on an assessment of where in the measurable CTS spectrum the $\alpha$-particles are going to generate most of the scattered radiation [26,27]. Thermal deuterium (and tritium) ions moving in parallel to $\mathbf{k}^{\delta}$ can have similar projected velocities as MeV-range $\alpha$-particles moving almost perpendicularly to $\mathbf{k}^{\delta}$. The projected velocity $u$ is proportional to the Doppler shift in the measured spectrum. The dense thermal bulk population can hence effectively mask the fairly dilute energetic $\alpha$-particle population at low Doppler shifts. Here we estimate this minimum projected velocity, where the $\alpha$-particle signal starts to dominate, to be $u_{\alpha, \min } \sim 3 v_{t h, D}$ where $v_{t h, D}$ is the thermal velocity of deuterium. The factor three is not fixed but depends on the relative densities of the $\alpha$-particles and the bulk ions and how well we know the bulk plasma densities, temperatures, and drifts, in particular for deuterium due to its low mass. On the back-of-the-envelope, this leads to a minimum $\alpha$-particle energy

$$
E_{\alpha, \min } \sim \frac{m_{\alpha}}{m_{D}} \frac{u_{\alpha, \min }^{2}}{v_{t h, D}^{2}} T_{D} \sim 18 T_{D} \sim 300 \mathrm{keV} .
$$

for one particular pitch. The exact form of the region where we can hope to measure $\alpha$-particles by CTS is given by weight functions summarized in figure 5(c). For the CTS geometry at ITER, the minimum detectable $\alpha$-particle energy increases towards extreme pitches, such that $\alpha$-particles with $|p| \sim 0.9-1$ cannot be measured. To detect the $\alpha$-particles, a CTS with an oblique view would be required $\left(\phi \sim 0^{\circ}-50^{\circ}\right)$. The region with high gross velocity-space sensitivity is circumscribed by a narrow stripe of 
intermediate sensitivity where the $\alpha$-particles produce signals only on one side of the CTS spectrum due to the slight asymmetry of the CTS weight functions about $p=0$.

We now go one step further and illustrate the gross velocity-space sensitivity of the combined diagnostic set of CTS and GRS. Figure 6 is obtained by summing the weight functions of all spectral points normalized by their associated noise levels. As the noise levels are estimated on theoretical grounds, we should keep in mind that the amplitudes in figure 6 are uncertain. Nevertheless, it is an efficient illustration of the topology of the gross velocity-space sensitivity of the combined diagnostic set at ITER and it provides useful insight into the matrix $W$ required for velocity-space tomography (section 4). Salient features from the individual diagnostics can be recognized in figure 6 . $\alpha$-particles at energies below $\sim 0.3 \mathrm{MeV}$ and co- and counter-going $\alpha$-particles at extreme pitches $(|p| \gtrsim 0.9)$ and energies up to $\sim 1.7 \mathrm{MeV}$ can hardly be diagnosed by CTS or GRS. The gross velocity-space sensitivity increases substantially from about 1.7 MeV upwards and becomes excellent around the $2 \mathrm{MeV}$ resonance of the $4.44 \mathrm{MeV}$ $\gamma$-ray peak. For energies close to this resonance and upwards, the gross velocity-space coverage of the combined diagnostic set at ITER is good. However, CTS is practically the only diagnostic detecting $\alpha$-particles below $\sim 1.7 \mathrm{MeV}$, covering the pitch range of $|p| \lesssim 0.8-0.9$. This causes the protrusion below the $2 \mathrm{MeV}$ resonance of the $4.44 \mathrm{MeV}$ $\gamma$-ray peak. Overall, while figure 6 is an illustrative summary of the diagnosed velocity space at ITER, it does not reveal our incapability to determine the sign of the pitch of an ion.

\section{Velocity-space tomography based on GRS and CTS at ITER}

In this section we analyze the inference of the $\alpha$-particle distribution at ITER by standard velocity-space tomography using analytic test functions to reveal basic features of the tomography problem. Realistic $\alpha$-particle distributions computed by ASCOT will be studied in section 6 . In standard velocity-space tomography, we solve the Tikhonov problem

$$
\text { minimize }\left\|\left(\begin{array}{c}
W \\
\lambda L
\end{array}\right) F-\left(\begin{array}{c}
S \\
0
\end{array}\right)\right\|_{2}^{2} \quad \text { subject to } F \geq 0
$$

with the mathematically equivalent formulation

$$
\text { minimize }\left\{\|W F-S\|_{2}^{2}+\lambda^{2}\|L F\|_{2}^{2}\right\} \quad \text { subject to } \quad F \geq 0 .
$$

$W$ is a matrix consisting of weight functions, $S$ is the measured signal written as a vector, $L$ is a penalty matrix, and $F$ is the velocity distribution function rearranged as a vector [70]. In $0^{\text {th }}$-order Tikhonov regularization $L$ is the identity matrix penalizing large amplitudes of $F$. In $1^{\text {st }}$-order Tikhonov regularization $L$ is a numerical gradient operator penalizing large gradients [68]. The regularization parameter $\lambda$ balances the relative sizes of the data fitting residual $W F-S$ and the penalty term $L F$ and must be determined as part of the solution. An interpretation of what the solution $F$ to the Tikhonov problem represents for almost perpendicular diagnostic systems will be 
given in section 5 . Here we restrict our focus to finding $F$ by standard velocity-space tomography in a few examples.

Figure 7 shows our best attempt to infer the 2D classical $\alpha$-particle slowingdown distribution function by standard velocity-space tomography for energies down to $300 \mathrm{keV}$. The classical $\alpha$-particle slowing-down distribution (figure $7(\mathrm{a})$ ) is given by $[87,88]$

$$
f(E)=\frac{C E^{1 / 2}}{E^{3 / 2}+E_{c}^{3 / 2}} \operatorname{erfc}\left(\frac{E-E_{b}}{\Delta E}\right) .
$$

Here the birth energy is $E_{b}=3.5 \mathrm{MeV}$ and the crossover energy (where drag on ions equals drag on electrons) is $E_{c}=660 \mathrm{keV}$, and we use a width of $\Delta E=100 \mathrm{keV}$ in the argument of the complementary error function. $C$ is a constant set to obtain the desired total density. The slowing-down distribution is isotropic in velocity space so that it does not depend on the pitch. We calculate synthetic spectra based on this distribution function with the combined GRS and CTS diagnostic system, add $10 \%$ noise and calculate inversions of the noisy, synthetic spectra. The inversion in figure 7(b) resembles the original function only coarsely. The distribution decreases monotonically for increasing energies at a given pitch, and the phase-space density of ions above the birth energy of $3.5 \mathrm{MeV}$ is practically zero. However, the inversion is plagued by artifacts. The isotropy of the original function is lost in the inversion. The amplitudes of the inversion do depend on the pitch, such that a wave-like pattern is formed. The shape of the CTS weight functions appears to be imprinted in the reconstruction. The artifacts appear because CTS provides practically the only view below about $1.7 \mathrm{MeV}$ where the GRS measurements are only very weakly sensitive. This behaviour relates well to previous findings that inversions based on one view are plagued by artifacts for realistic noise levels [62].

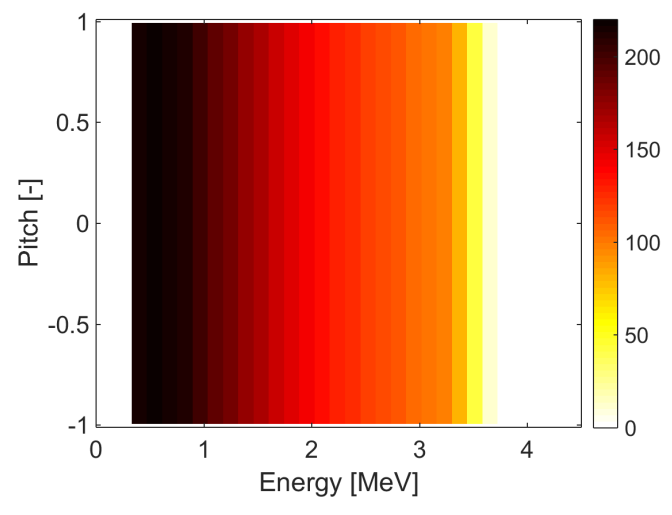

(a)

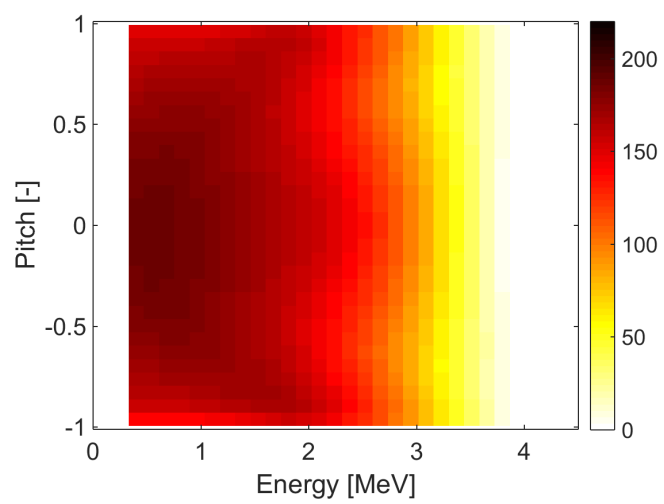

(b)

Figure 7. (a) Classical slowing-down distribution in units $\left[10^{10} /\left(\mathrm{m}^{3} \mathrm{eV}\right)\right]$. (b) Tomographic inversion down to $300 \mathrm{keV}$ from noisy, synthetic GRS/CTS measurements based on the distribution in (a). The inversion is plagued by artifacts since CTS is effectively the only available fast-ion diagnostic in the range $300 \mathrm{keV}$ to $1.7 \mathrm{MeV}$.

A strategy to circumvent these artifacts is to restrict the velocity-space region to 
energies larger than $1.7 \mathrm{MeV}$, so that more than one diagnostic is available everywhere in velocity space. Hence the CTS measurements that have significant sensitivity to $\alpha$ particles below $1.7 \mathrm{MeV}$ are not used in the tomography problem (see figure 6). The CTS measurements without sensitivity below $1.7 \mathrm{MeV}$ and the GRS measurements can then be used to reconstruct the velocity space above $1.7 \mathrm{MeV}$.

Reconstructions restricted to energies above $1.7 \mathrm{MeV}$ are presented in figure 8 . Figure 8(a) and (b) show the isotropic $\alpha$-particle slowing-down distribution function and its reconstruction above $1.7 \mathrm{MeV}$. They are in excellent agreement. The decreasing density towards higher energies is well captured, as is the birth energy at $3.5 \mathrm{MeV}$. The reconstruction is isotropic to a high degree in agreement with the original function. This demonstrates that the classical, isotropic slowing-down distribution can be reconstructed with the diagnostics currently planned at ITER in the region above $1.7 \mathrm{MeV}$ where both CTS and GRS are sensitive.

In figure 8(c) and (d) we reconstruct an anisotropic function with symmetry about $p=0$. The anisotropic function has decreasing phase-space densities towards pitches of $p \sim \pm 1$. This type of distribution function could be a model for the $\alpha$-particles after a sawtooth crash that has ejected predominantly passing particles rather than trapped particles. This type of anisotropy with symmetry in the co-going and counter-going particles is also captured well in the reconstruction.

However, in figure 8(e) and (f) we attempt to reconstruct a distribution function that is asymmetric about $p=0$. The original function is lopsided towards negative pitches as a model for ejection of co-going particles from the classical slowingdown distribution. This asymmetry is poorly captured in the reconstruction. The reconstruction is only marginally lopsided towards negative pitches and is almost symmetric such that it erroneously also shows ejection of counter-going particles. The failure to reconstruct the asymmetric distribution function originates from the very poor sensitivity to the sign of the pitch of the current set of diagnostics. We will discuss velocity-space tomography techniques for perpendicular and almost perpendicular systems in section 5 .

We could distinguish co- and counter-going ions and the sign of the pitch if ITER had a tangential or oblique fast-ion diagnostic view with respect to the magnetic field. Figure 9 illustrates weight functions for an oblique GRS view with a viewing angle of $\phi=30^{\circ}$ which is similar to that currently being installed at JET. Typical weight functions are asymmetric about $p=0$ allowing the reconstruction of asymmetric functions. At ITER such an oblique GRS instrument could be installed in any port plug from a physics point of view, but this is often challenging from an engineering point of view. One option could be to place it in equatorial port plug 8 .

Figure 10 shows reconstructions of the asymmetric $\alpha$-particle distribution function from figure 8(e) assuming an additional oblique GRS view. The reconstruction captures the asymmetry and shape of the original function. This demonstrates that an oblique GRS view will be required, if we wish to be able to determine the sign of the pitch and tell co- and counter-going ions apart. Close inspection still reveals some erroneous 


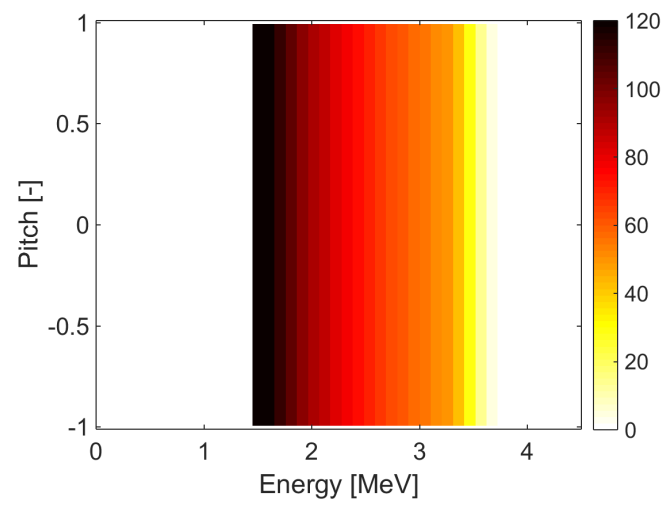

(a)

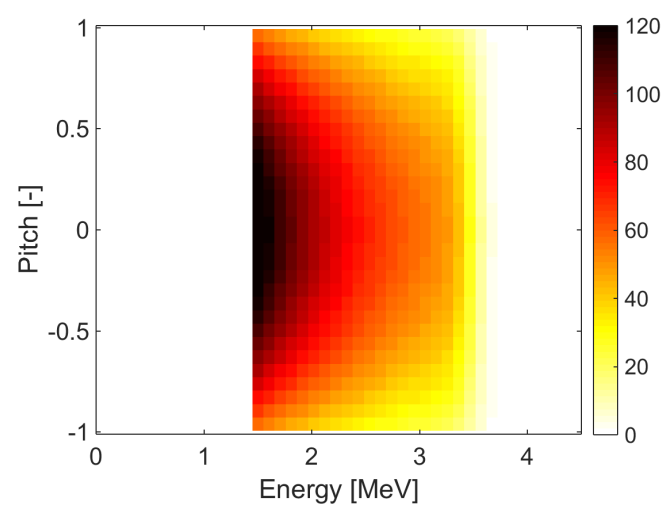

(c)

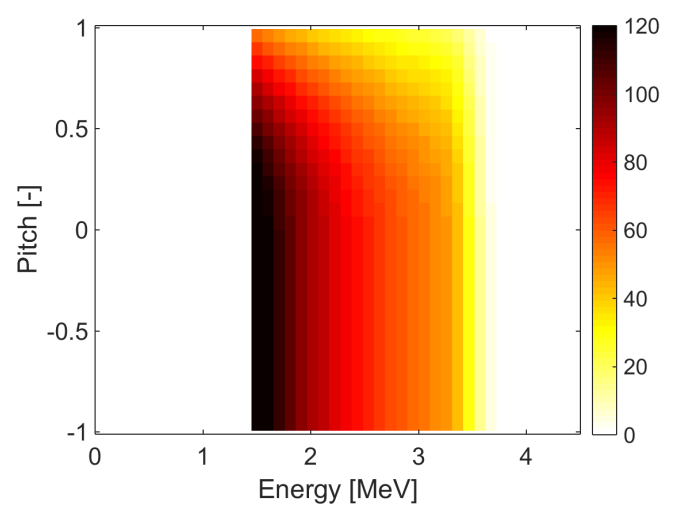

(e)

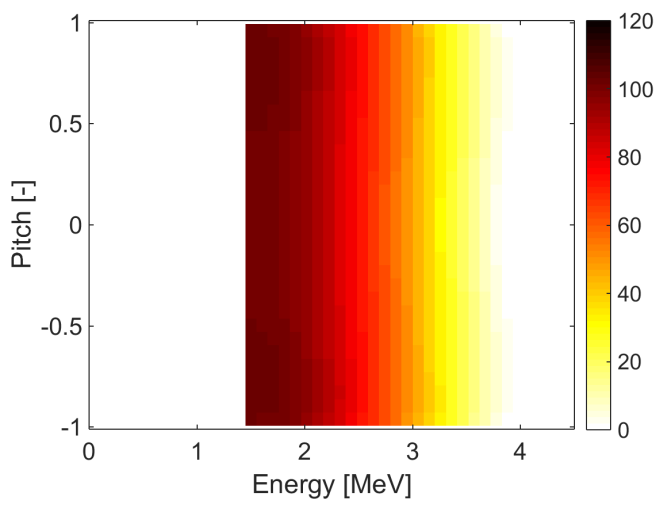

(b)

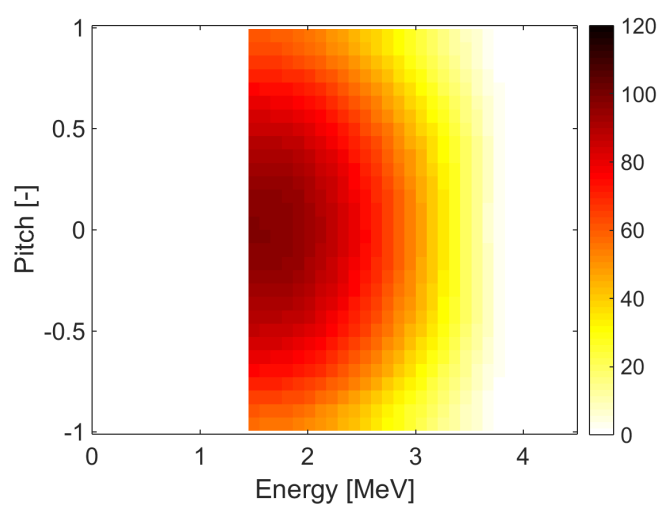

(d)

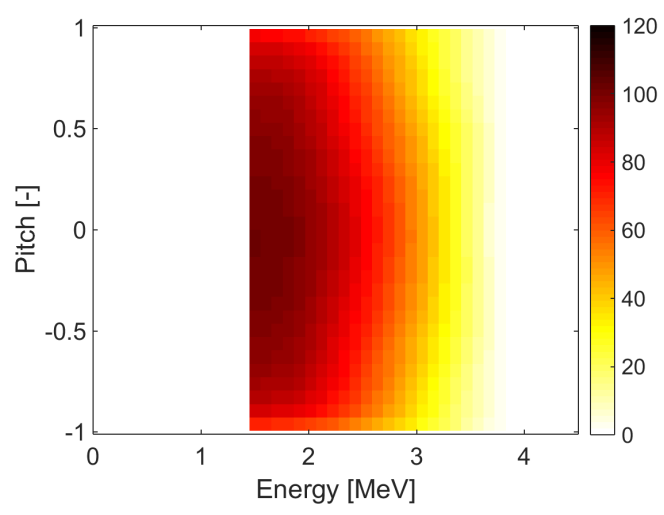

(f)

Figure 8. Test distribution functions in units $\left[10^{10} /\left(\mathrm{m}^{3} \mathrm{eV}\right)\right]$ and their respective tomographic inversions down to $1.7 \mathrm{MeV}$ from noisy synthetic data. (a) Isotropic test function. (b) Inversion based on (a). (c) Anisotropic test function with symmetry about $p=0$. (d) Inversion based on (c). (e) Anisotropic test function with aymmetry about $p=0$. (f) Inversion based on (e). 


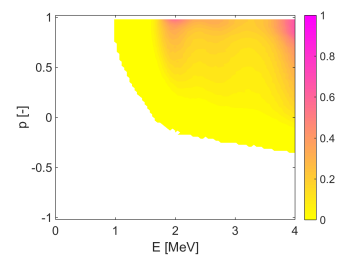

(a) $\Delta E_{\gamma}=-60 \mathrm{keV}$

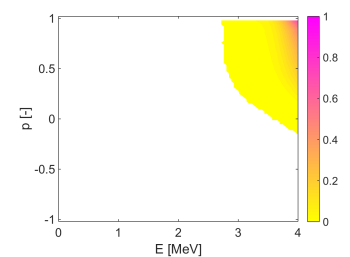

(e) $\Delta E_{\gamma}=-40 \mathrm{keV}$

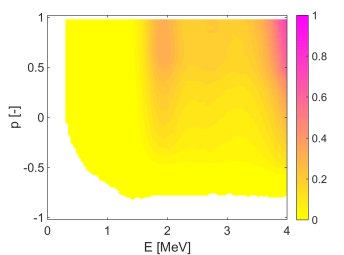

(b) $\Delta E_{\gamma}=-30 \mathrm{keV}$

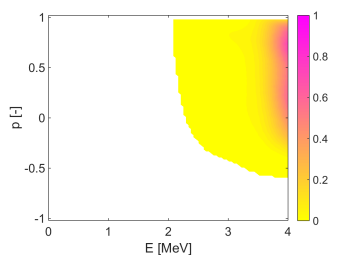

(f) $\Delta E_{\gamma}=-20 \mathrm{keV}$

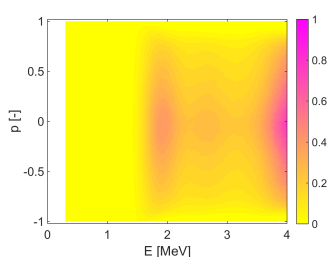

(c) $\Delta E_{\gamma}=0 \mathrm{keV}$

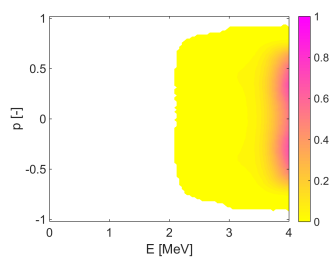

(g) $\Delta E_{\gamma}=0 \mathrm{keV}$

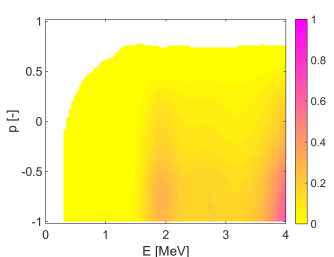

(d) $\Delta E_{\gamma}=+30 \mathrm{keV}$

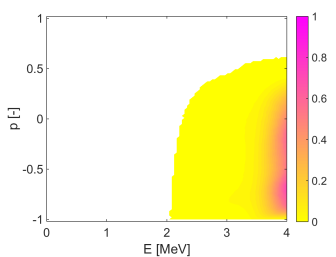

(h) $\Delta E_{\gamma}=20 \mathrm{keV}$

Figure 9. GRS weight functions at various Doppler shifts $\Delta E_{\gamma}$ for proposed extra diagnostic views at $\phi=30^{\circ}$. Upper row: $4.44 \mathrm{MeV}$ peak. Lower row: $3.2 \mathrm{MeV}$ peak.

depletion at negative pitches in the inversion, but this artifact is strongly suppressed compared with $8(\mathrm{f})$.

To quantify the improvement due to the additional oblique GRS view, we compute the partial fast-ion densities for ions in the upper and lower $(E, p)$-halfplanes corresponding to positive and negative pitches for the inversion and the true solution (table 1). The inversions generally have similar $\alpha$-particle densities as the corresponding true solutions. However, solutions obtained with the currently planned set of GRS/CTS diagnostics always give symmetric distributions of $\alpha$-particles, despite any asymmetry in the true solution. The asymmetry in the true distribution is captured much better with an additional oblique GRS view.

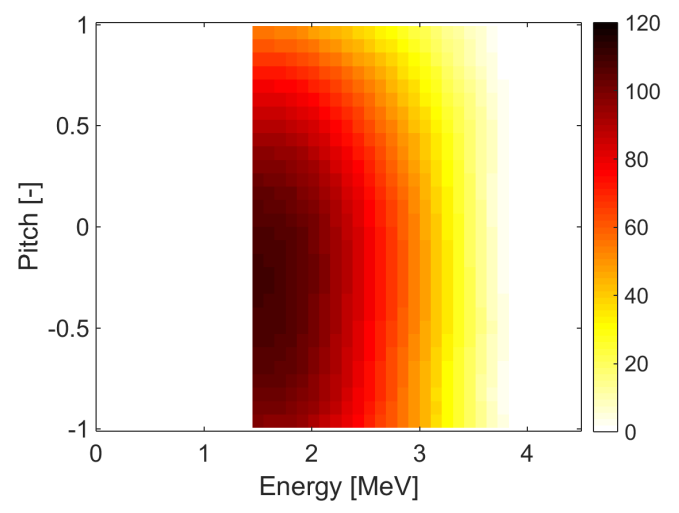

Figure 10. Reconstruction of the asymmetric $\alpha$-particle distribution function from figure 8 (e) with an additional oblique GRS view at $\phi=30^{\circ}$. 
Table 1. $\alpha$-particle densities $n_{\alpha}\left[10^{18} / \mathrm{m}^{3}\right]$ for $E_{\alpha}>1.7 \mathrm{MeV} . n_{\alpha}^{+}$and $n_{\alpha}^{-}$are the densities for positive and negative pitch, respectively. Their ratio $n_{\alpha}^{+} / n_{\alpha}^{-}$measures the asymmetry of the distribution function.

\begin{tabular}{l|ll|ll|lll}
\hline Figure & $8(\mathrm{a})$ & $8(\mathrm{~b})$ & $8(\mathrm{c})$ & $8(\mathrm{~d})$ & $8(\mathrm{e})$ & $8(\mathrm{f})$ & 10 \\
\hline$n_{\alpha}$ & 2.93 & 2.76 & 2.39 & 2.29 & 2.66 & 2.57 & 2.58 \\
$n_{\alpha}^{+}$ & 1.46 & 1.37 & 1.19 & 1.15 & 1.19 & 1.28 & 1.16 \\
$n_{\alpha}^{-}$ & 1.46 & 1.38 & 1.19 & 1.14 & 1.46 & 1.29 & 1.43 \\
\hline$n_{\alpha}^{+} / n_{\alpha}^{-}$ & 1.00 & 0.99 & 1.00 & 1.01 & 0.82 & 0.99 & 0.81 \\
\hline
\end{tabular}

\section{Velocity-space tomography for diagnostics with perpendicular and almost perpendicular views}

In this section we discuss the implications of the almost perpendicular viewing directions of the fast-ion diagnostics planned for ITER. Assume first a completely perpendicular system as an idealized model. In this case there is no way to infer the full velocity distribution function $F$ as we never know if a given ion has a pitch of $p$ or $-p$. It is not even possible to infer a pixel function $F_{i}$ consisting of just one pixel $i$. The measured signal for the pixel function is as usual

$$
S_{i}=W F_{i} .
$$

As we are not sensitive to the sign of the pitch, the mirror image of $F_{i}, \bar{F}_{i}$, produces the same signal as $F_{i}$ :

$$
S_{i}=W \bar{F}_{i} .
$$

The bar denotes the mirroring operation about $p=0$. Adding $n$ times equation 6 to $m$ times equation 7 and solving for $S$ gives

$$
S_{i}=\frac{n}{m+n} W F_{i}+\frac{m}{m+n} W \bar{F}_{i} .
$$

We introduce the function $F_{i, m i x}$ as a mixture of $F_{i}$ and its mirror image $\bar{F}_{i}$ :

$$
F_{i, m i x}=\frac{n}{m+n} F_{i}+\frac{m}{m+n} \bar{F}_{i} .
$$

The measurable signal for $F_{i, m i x}$ is

$$
S_{i}=W F_{i, m i x} .
$$

which is the same as for $F_{i}$ and $\bar{F}_{i}$. If $F_{i}$ and $\bar{F}_{i}$ produce the same signal, then so does any mix of $F_{i}$ and $\bar{F}_{i}, F_{i, m i x}$, constructed according to equation 9 . This demonstrates our incapability to tell the sign of the pitch apart for entirely perpendicular systems. This result also holds for arbitrary distribution functions since these can be constructed from pixel functions:

$$
F=\sum_{i} F_{i}
$$


The total signal for an arbitrary distribution function can also be constructed as the sum of signal for pixel functions:

$$
S=\sum_{i} S_{i}=\sum_{i} W F_{i, m i x}=W \sum_{i} F_{i, m i x}
$$

Hence we obtain

$$
S=W F_{m i x}
$$

showing that any mix of $F$ and $\bar{F}$ gives the same signal. For a completely perpendicular system, we can hence infer $F_{\text {mix }}$ from a measured signal by velocity-space tomography. $F_{\text {mix }}$ still holds much information about $F$ but we do not make any statement about how the phase-space densities are shared among $F$ and $\bar{F}$ for each pixel according to equation 9 . Hence only $f(E,|p|)$ can be inferred by velocity-space tomography for completely perpendicular systems.

Solutions found by Tikhonov regularization tend to be close to up-down symmetric about $p=0$ for completely perpendicular systems. This is not surprising as all weight functions are up-down symmetric about $p=0$ in this case. However, it is also instructive to consider the symmetry in terms of the Tikhonov problem. Any mix $F_{m i x}$ has the same residual $W F_{m i x}-S$ as $F$, and hence the least-squares term $\|W F-S\|_{2}^{2}$ of Tikhonov's minimization problem (equation 4 ) is not able to separate these. Nevertheless, the Tikhonov penalty term $\left\|L F_{\text {mix }}\right\|_{2}^{2}=\left\|\frac{n}{m+n} L F+\frac{m}{m+n} L \bar{F}\right\|_{2}^{2}$ in equation 4 is smallest for a mix (equation 9) with $m=n$, i.e., when the mix is symmetric (this follows from a bit of algebra omitted here). Therefore the Tikhonov regularization will select for solutions that are close to up-down symmetric. The up-down symmetric solution is obtained for $m=n=1$ in equation 9 :

$$
F_{u d}=\frac{1}{2}(F+\bar{F}) \text {. }
$$

To directly solve for this up-down symmetric solution and explicitly incorporate our inability to determine the sign of the pitch in the Tikhonov problem, we can pose the problem by computing the up-down symmetric $F_{u d}$ in one halfplane only, e.g. the halfplane with $p>0$. The signal due to $F_{u d}$ in the full velocity space is

$$
S=W F_{u d}
$$

We introduce the parts of the weight functions with positive pitch, $W^{+}$, and the parts with negative pitch, $W^{-}$, and similarly for $F_{u d}, F_{u d}^{+}$and $F_{u d}^{-}$. Then we can write the signal as

$$
S=W^{+} F_{u d}^{+}+W^{-} F_{u d}^{-} .
$$

Without changing the result, we can mirror $W^{-}$and $F_{u d}^{-}$into the upper halfplane and compute the signal as

$$
S=W^{+} F_{u d}^{+}+\bar{W}^{-} \bar{F}_{u d}^{-} .
$$

Since we are assuming an up-down symmetric function $F_{u d}$, we have

$$
\bar{F}_{u d}^{-}=F_{u d}^{+} \text {. }
$$


Hence

$$
S=\left(W^{+}+\bar{W}^{-}\right) F_{u d}^{+}
$$

For exactly $\phi=90^{\circ}$, we also have $W^{+}=\bar{W}^{-}$. For the almost perpendicular system at ITER ( $\phi \sim 90^{\circ}$, but not exactly), $F_{u d}$ is a good approximation to the computed $F$ and illustrates our limitation to infer the pitch. In this case we can simply use equation 19 to infer $F_{u d}^{+}$with the explicit prior information of up-down symmetry. This conveniently uses half the number of grid points but a small error is introduced.

Asymmetries in the original function are not captured by the inversion and are instead redistributed towards a more symmetric inversion as illustrated in figure $8(\mathrm{f})$. We can still make statements about the $2 \mathrm{D}$ velocity distribution function $f(E,|p|)$, just not about the sign of the pitch. We can gain insight into the solution obtained using $1^{\text {st }}$-order Tikhonov regularization by the generalized singular value decomposition (GSVD) (see [89] and the references therein). It provides the basis of the solutions allowed by the weight matrix $W$ and the regularization matrix $L$ in the Tikhonov problem. As explained in [89], the Tikhonov solution is expressed in terms of the GSVD basis functions, and it can be shown that the Tikhonov solution is dominated by those components that correspond to the largest generalized singular values. The reconstruction of asymmetric Tikhonov solutions from noisy data thus requires that some of the dominant generalized singular values are associated with asymmetric basis functions. Figure 11 illustrates the nine most important basis functions for the currently planned set of $\alpha$-particle diagnostics and for the enhanced set with an extra GRS view at $\phi=30^{\circ}$. The first eight basis functions of the Tikhonov solution for the currently planned set are almost up-down symmetric which results in almost up-down symmetric solutions. On the contrary, several of the nine dominant basis functions for the enhanced set are up-down asymmetric such that it is possible to compute up-down asymmetric solutions.
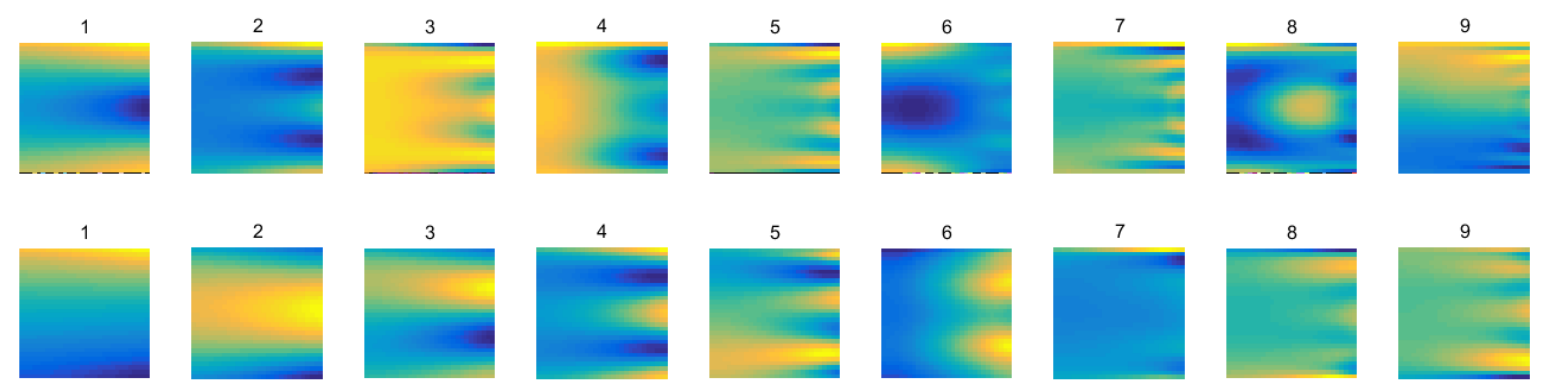

Figure 11. The nine dominant basis functions of a GSVD (yellow: positive, blue: negative). Upper row: The currently planned set of $\alpha$-particle diagnostics where the first eight basis functions are up-down symmetric. Lower row: The enhanced system with an additional GRS view at $\phi=30^{\circ}$. In this cases several of the first nine basis functions are asymmetric and thus allow the construction of asymmetric regularized solutions.

We can consider the Tikhonov problem to devise a strategy to allow asymmetric regularized solutions. Any asymmetry in the inversion needs to have an incentive in 
the measurements, which is, however, very hard to deliver with almost perpendicularly viewing diagnostics. In the Tikhonov problem, the upper row $W F-S$ can introduce asymmetry. The signal is

$$
S=W F+\epsilon
$$

where $\epsilon$ is the noise. Hence the residuals of the true solution $F$ and an erroneous solution $F_{m i x}$, in which the mirror image of the true solution appears, are, respectively,

$$
\begin{aligned}
& r=|S-W F|=\epsilon \\
& r_{\text {mix }}=\left|S-W F_{\text {mix }}\right|=\left|W\left(F-F_{\text {mix }}\right)+\epsilon\right|
\end{aligned}
$$

The three competing terms determining the degree of asymmetry are $\epsilon, W\left(F-F_{\text {mix }}\right)$ and $L F_{m i x}$. The term $W\left(F-F_{m i x}\right)$ can introduce asymmetry in this case and will effectively prevent the appearance of $\bar{F}$ in the inversion measurements if measurements in tangential or oblique views are made (and if the true $F$ is indeed up-down asymmetric). However, for almost perpendicular views $W\left(F-F_{\text {mix }}\right)$ is small and it competes with the Tikhonov penalty term $\lambda L F_{m i x}$ which will tend to promote evenly distributed or smooth solutions for $0^{t h}$ - and $1^{\text {st }}$-order Tikhonov regularization, respectively. For almost perpendicular systems this will tend to promote the appearance of $\bar{F}$ in the solution which also explains the data fairly well. We found in section 4 that $F_{\text {mix }}$ does not have large enough residuals for the almost perpendicular CTS view (and not at all by GRS) to prevent the appearance of $\bar{F}$ in the solution.

Hence our inversions in section 4 should be understood to represent $F_{\text {mix }}$ rather than $F$, i.e. the amplitude in a given pixel represents a weighted sum of the amplitudes of the pixel and its mirror image. To become more sensitive to the sign of the pitch, the term $W\left(F-F_{\text {mix }}\right)$ must be increased. This could in principle be done by higher-signalto-noise ratio measurements but we do not regard this as realistic [62]. The only way appears to be an extra oblique GRS view which leads to large $W\left(F-F_{\text {mix }}\right)$ for realistic noise levels.

\section{Reconstruction of ASCOT simulations based on GRS and CTS}

The classical $\alpha$-particle slowing-down distribution is symmetric. However, several effects can lead to asymmetry and anisotropy in the $\alpha$-particle distribution, which we discuss here and in the next section. Here we assess how much anisotropy and asymmetry is expected in ITER based on neoclassical theory. The Monte Carlo orbit-following code ASCOT [90] is used for studies of neoclassical transport of minority particles in toroidal magnetic fusion devices. It follows guiding-center- and gyro-orbits of charged particles in realistic geometries. The particles are represented by weighted markers initialized to represent the source population of interest. ASCOT simulates collisional interactions with an assumed Maxwellian background by the Monte Carlo method using given temperature and density profiles.

ASCOT has recently been applied to extensive studies of fusion $\alpha$-particle wall

loads in various ITER scenarios $[91,92]$ with three-dimensional magnetic background 
and first wall. The effects of toroidal field ripple, mitigated by ferritic inserts at the toroidal field coils, as well as non-periodic magnetic field perturbations caused by the ferritic test blanket modules and control coils for edge-localized modes were studied. In the simulations, an isotropic $\alpha$-particle source corresponding to the local DT fusion reaction density of each scenario was assumed, and 100.000 weighted $\alpha$-markers were followed from their birth energy down to $50 \mathrm{keV}$.

In addition to the wall loads that were the primary goal of the simulations previously described [91,92], the four-dimensional slowing-down distribution $f(R, z, p, E)$ was recorded for the $\alpha$-population for the ITER baseline (15 MA) and the ITER hybrid (12.5 MA) scenarios in the absence of non-periodic magnetic perturbations. We study these distributions as more realistic models for the $\alpha$-particles which include anisotropy due to neoclassical transport.

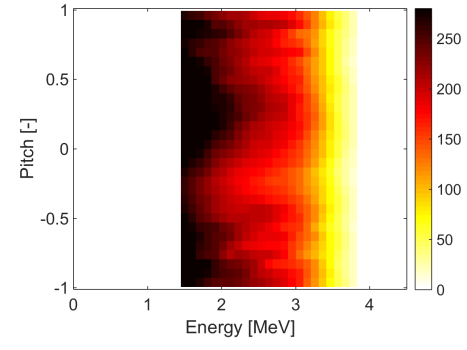

(a)

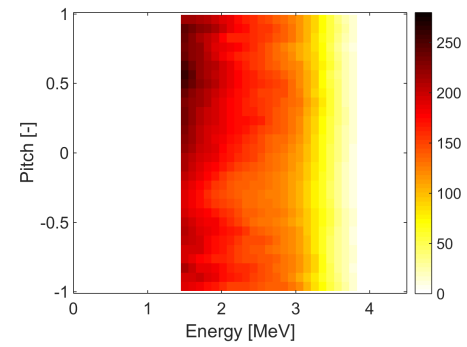

(d)

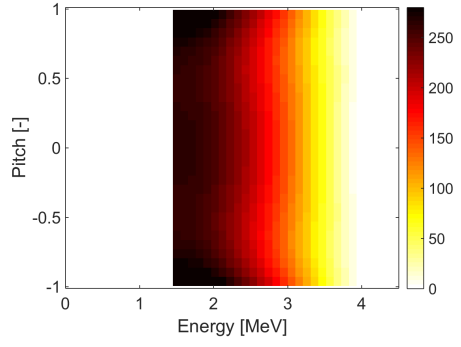

(b)

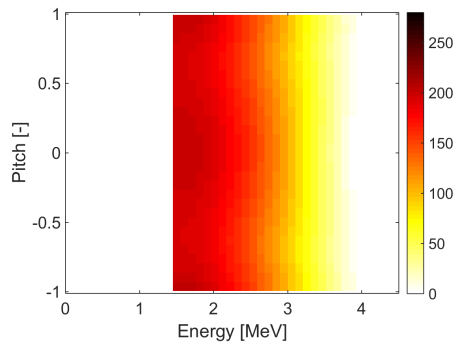

(e)

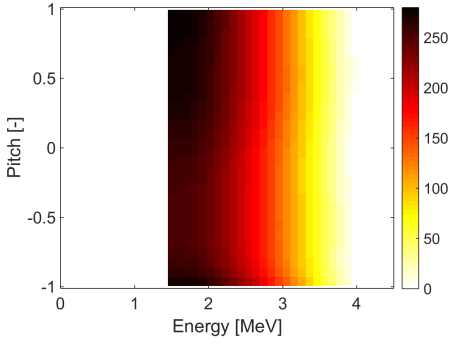

(c)

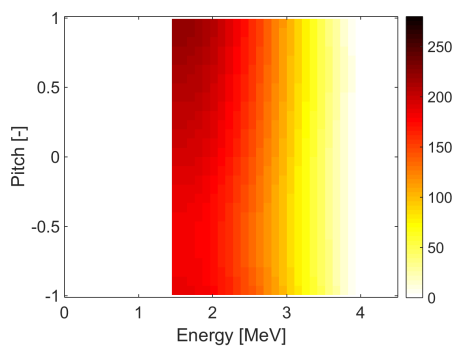

(f)

Figure 12. Reconstructions of $\alpha$-particle distribution functions from ASCOT. (a) ITER baseline scenario. (b) Reconstruction of baseline scenario with planned GRS/CTS diagnostics. (c) As (b) with an extra $30^{\circ}$ GRS detector. (d) ITER hybrid scenario. (e) Reconstruction of hybrid scenario with planned GRS/CTS diagnostics. (f) As (e) with an extra $30^{\circ}$ GRS detector.

Figure 12 illustrates $\alpha$-particle distribution functions for the ITER baseline and the ITER hybrid scenarios. Reconstructions from noisy, synthetic measurements based on these are illustrated for the currently planned set of diagnostics and for our proposed extra oblique GRS view at $30^{\circ}$.

Neoclassically, the $\alpha$-particle distribution is anisotropic even for an isotropic $\alpha$ particle birth velocity distribution due to the different orbit topologies. Passing particles can either have parallel velocities aligned with the plasma current (positive pitch, cogoing), or they have parallel velocities against the current direction (negative pitch, 
Table 2. $\alpha$-particle densities $n_{\alpha}\left[10^{18} / \mathrm{m}^{3}\right]$ for $E_{\alpha}>1.5 \mathrm{MeV} . n_{\alpha}^{+}$and $n_{\alpha}^{-}$are the densities for positive and negative pitch, respectively. Their ratio $n_{\alpha}^{+} / n_{\alpha}^{-}$measures the asymmetry of the distribution functions.

\begin{tabular}{l|lll|lll}
\hline Scenario & Baseline & \multicolumn{5}{|l}{ Hybrid } \\
\hline & ASCOT & GRS/CTS & Extra GRS & ASCOT & GRS/CTS & Extra GRS \\
\hline$n_{\alpha}$ & 7.76 & 7.55 & 7.54 & 5.71 & 5.58 & 5.60 \\
$n_{\alpha}^{+}$ & 4.06 & 3.78 & 3.90 & 3.03 & 2.80 & 2.97 \\
$n_{\alpha}^{-}$ & 3.70 & 3.77 & 3.64 & 2.68 & 2.78 & 2.63 \\
\hline$n_{\alpha}^{+} / n_{\alpha}^{-}$ & 1.10 & 1.00 & 1.07 & 1.13 & 1.01 & 1.13 \\
\hline
\end{tabular}

counter-going). There are more particles born on the outboard (low-field) side as compared with the inboard (high-field) side due to the larger volume. A co-going particle on the outboard midplane drifts first inwards towards the plasma center and then outwards back to its starting flux surface. On the contrary, a counter-going particle drifts first outwards and then inwards back to the starting flux surface. The slowingdown times tend to be larger on the inner flux surfaces traversed by the co-going particles due to the larger temperatures as compared to the outer flux surfaces traversed by the counter-going particles. The steady-state $\alpha$-particle distribution is therefore expected to be biased towards co-going particles. Similarly, a trapped $\alpha$-particle with positive pitch on the outboard midplane is on the outer leg of the banana orbit whereas with negative pitch it is on the inner leg. ASCOT simulations contain this type of asymmetry.

As expected, this asymmetry cannot be captured for the currently planned GRS/CTS diagnostic. Our proposed additional $30^{\circ} \mathrm{GRS}$ view substantially improves the diagnostic of this asymmetry which is only just apparent in figure 12. Nevertheless, the improvement is quantified in table 2 showing the partial $\alpha$-particle densities with positive and negative pitches and their ratios.

If an additional $30^{\circ}$ GRS view cannot be made available, we instead need to be aware that our computed solution is a mix of the solution and its mirror image. In this case it could be misleading to plot the entire function $f(E, p)$. Instead, we propose to present our results in terms of the distribution function $f(E,|p|)$ which is not sensitive to the sign of the pitch. Figure 13 illustrates such halfplane reconstructions for the baseline and the hybrid scenarios without our proposed additional $30^{\circ} \mathrm{GRS}$ view. We define the function $f(E,|p|)=f(E, p)+\bar{f}(E, p)$ for $p=[0 ; 1]$. The distribution $f(E,|p|)$ is computed in two ways: Firstly, we infer the full velocity-space tomography formalism and compute $f(E,|p|)$ afterwards. Secondly, we directly infer $f(E,|p|)$ assuming updown symmetry. As expected, the two methods give similar results in good agreement with the true solution. 


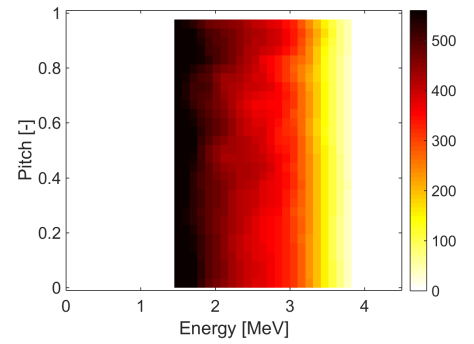

(a)

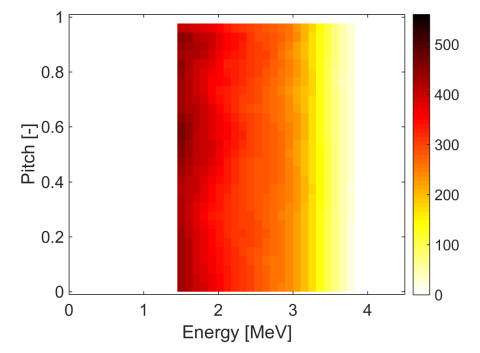

(d)

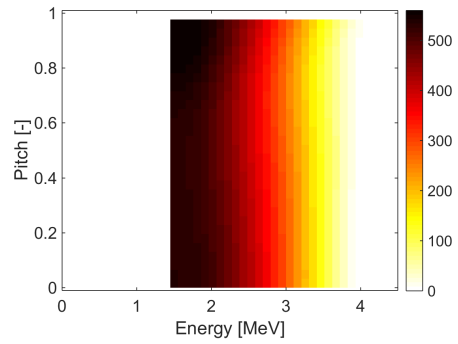

(b)

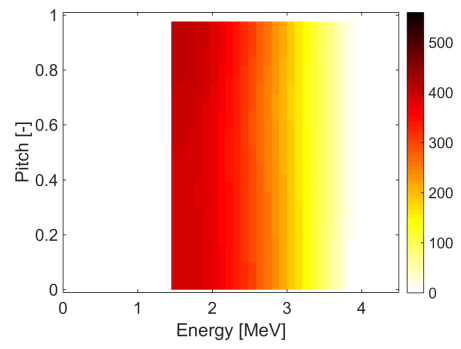

(e)

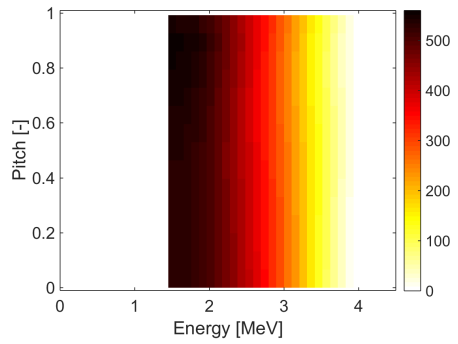

(c)

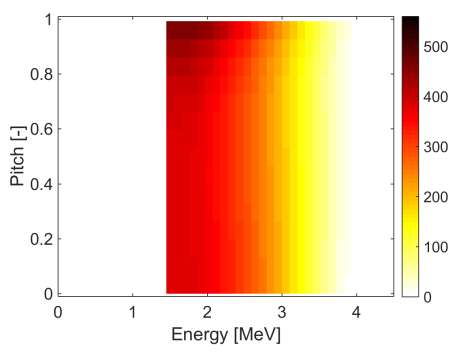

(f)

Figure 13. Reconstructions of sign-insensitive $\alpha$-particle distribution functions $f(E,|p|)$ from ASCOT. (a) ITER baseline scenario represented as $f(E,|p|)$ (b) Full reconstruction of baseline scenario represented as $f(E,|p|)$. (c) Direct reconstruction of $f(E,|p|)$. (d)-(f) As for (a)-(c), but for the ITER hybrid scenario.

\section{Sources of anisotropy in the $\alpha$-particle distribution}

As discussed in section 6 , the $\alpha$-particle distribution is anisotropic even for an isotropic birth velocity distribution in neoclassical transport theory due to the different orbit topologies. This type of asymmetry is reproduced in our ASCOT simulation which showed $n_{\alpha}^{+} / n_{\alpha}^{-} \sim 1.10$ or larger. In this section we discuss two additional sources of asymmetry.

A second source of asymmetry in the $\alpha$-distribution is that the birth profile of the $\alpha$-particles is not isotropic. As $\alpha$-particles are born in the $\mathrm{D}(\mathrm{T}, \mathrm{n}) \alpha$ reaction, anisotropies in particular in the deuterium velocity distribution due to NBI lead to anisotropies in the $\alpha$-particle birth profile. The $\alpha$-distribution will therefore be lopsided towards the direction of the NBI injection, as the suprathermal NBI ions are much more likely to undergo a fusion reaction than the bulk deuterium population. Any rotation induced by the torque of the beam enhances this effect. The ASCOT simulation assumes an isotropic birth profile, and so our simulations do not account for this effect. As the NBI ions are injected with positive pitch, this bias is expected to enhance the asymmetry due to the drift orbits as computed in the ASCOT simulation.

A third source of asymmetry is the physics of wave-particle interaction between energetic particles and Alfvén eigenmodes which is also sensitive to the sign of the pitch of the particle motion. This sensitivity determines the type of instabilities observed and the class of particles which undergo wave-induced transport resulting in anisotropy. 
To study the drive of eigenmodes, we consider the distribution function $F\left(E, \mu, P_{\zeta}\right)$ in constants-of-motion space. $E$ is the energy, $\mu$ the magnetic moment and $P_{\zeta}$ the toroidal canonical angular momentum respectively given by

$$
E=\frac{1}{2} m v^{2}, \mu=\frac{m v_{\perp}^{2}}{2 B}, P_{\zeta}=m R v_{\zeta}-q \Psi
$$

where $\Psi$ is the poloidal magnetic flux. When energetic particle velocities are comparable to the phase velocity $v_{A}$ of the Alfvén wave

$$
\frac{\omega}{k} \approx v_{A}=\frac{B}{\sqrt{\mu_{0} \rho}}
$$

particles resonantly exchange energy with the waves. $\omega$ is the mode frequency, $k$ is the wave number, $B$ is the magnetic field, $\mu_{0}$ the vacuum permeability, and $\rho$ is the plasma density. Gradients in the distribution function at resonance can provide free energy to drive the modes $[93,94]$. The gradient in $\mu$ is not relevant as the ion cyclotron frequency exceeds the mode frequency by far so that $\mu$ is conserved. For a mode to be unstable, the drive must exceed the damping rate. Gradients in energy are usually negative $(\partial F / \partial E<0)$ whereas the mode frequency $\omega$ is positive. Therefore the growth rate associated with gradients in energy is usually negative, which damps the wave:

$$
\gamma_{L} \propto \omega \frac{\partial F}{\partial E}
$$

Spatial radial gradients enter $P_{\zeta}$, and they can drive an eigenmode given with the growth rate

$$
\gamma_{L} \propto n \frac{\partial F}{\partial P_{\zeta}}
$$

for a given toroidal mode number $n$ (see for example [93] equation 76). For drive of the mode, the sign of the radial gradient $\partial F / \partial P_{\zeta}$ matches the sign of the toroidal mode number $n$. As usually $\partial F / \partial P_{\zeta}>0$, positive mode numbers can be driven unstable. (A reversal in sign can suggest that gradients have reversed and that fast particle distributions have become hollow.) The sign of $n$ defines the direction of propagation of the corresponding shear Alfvén wave through the sign of [88]

$$
k_{\|} \approx \frac{1}{B}\left(\frac{m}{r} B_{\theta}+\frac{n}{R} B_{\phi}\right) .
$$

$k_{\|}$is the parallel wave number, $m$ is the poloidal mode number, $r$ and $R$ are the minor and major radii, and $B_{\theta}$ and $B_{\phi}$ are the poloidal and toroidal magnetic field. When waves are driven in this manner, they interact most strongly with particles travelling with the same speed and direction given by the resonance condition

$$
k_{\|} v_{\|}-\omega=0 .
$$

If a single mode interacts with a particle, the quantity $E-(\omega / n) P_{\zeta}$ is a constant of the motion, and so the particle changes its energy and toroidal canonical angular momentum according to

$$
n \Delta E=\omega \Delta P_{\zeta}
$$


which means that loss in energy to the wave leads to outward energetic particle transport towards the edge. The selectivity of the shear Alfvén waves in $v_{\|}$will thus induce an anisotropy in the $\alpha$-particle distribution function.

The current set of fast-ion diagnostics is not sensitive to the sign of the pitch, and the consequences of the three sources of asymmetry can therefore not be measured. Given the importance of understanding the $\alpha$-particle distribution function in ITER, we therefore propose that an additional GRS detector with an oblique viewing angle should be installed in ITER. With this additional GRS detector, these asymmetries in the $\alpha$-particle distribution can be tracked. This can give important clues on the physics of wave-particle interaction and the anisotropy of the $\alpha$-particle distribution function.

\section{Measurements of energy spectra for isotropic distributions}

Below $\alpha$-particle energies of $1.7 \mathrm{MeV}$, CTS is practically the only available diagnostic for confined $\alpha$-particles. With only one available view, reconstructions of the $\alpha$ particle distribution function below $1.7 \mathrm{MeV}$ has strong artifacts if standard velocityspace tomography methods are used. The ITER measurement requirements entail measurements of the $\alpha$-particle energy spectrum and density [77]. The $\alpha$-particle density could be estimated by assuming a functional form of the $\alpha$-particle distribution function, e.g. a slowing-down distribution, and fitting the spectra. However, velocity-space tomography is the only known way to measure the energy spectrum.

Here we present a method to nevertheless measure the energy spectrum even if only one view is available. To make up for the lack of measurements, additional prior information is used. We assume the velocity distribution function to be isotropic which is in contrast to the asymmetries observed in sections 6 and 7. A second assumption is that the measurable CTS signal due to NBI ions is negligible compared with measureable CTS signal due to $\alpha$-particles [26]. If we are prepared to neglect any asymmetry and CTS due to NBI ions, we can encode the isotropy assumption into the first-order Tikhonov regularization which penalizes large gradients in the velocity-distribution function. A solution with a high degree of isotropy can be selected for by penalizing derivatives in

pitch direction much more than derivatives in energy direction. Instead of the standard Tikhonov problem solving for a 2D velocity distribution function, we solve

$$
\text { minimize }\left\|\left(\begin{array}{c}
W \\
\lambda_{E} L_{E} \\
\lambda_{p} L_{p}
\end{array}\right) F-\left(\begin{array}{c}
S \\
0 \\
0
\end{array}\right)\right\|_{2} \quad \begin{array}{cc}
\text { subject to } & F \geq 0 \\
\lambda_{p} \gg \lambda_{E}
\end{array}
$$

which selects for isotropic solutions. Figure 14(a) presents an isotropic inversion where we have chosen $\lambda_{p}=100 \lambda_{E}$. The true solution is the $\alpha$-particle slowing-down distribution function from figure 7 (a). The inversion is in excellent agreement with the true solution which is expected since our assumption of isotropy as additional prior information is completely true in this case. However, any anisotropy such as those 
studied in figure 8 will not be captured with this approach as then the prior information is wrong. In fact, it may not be appropriate to characterize an anisotropic function by an energy spectrum since this parameter disregards any pitch dependence. We compare the energy spectrum of the original function used to compute the synthetic measurements with the energy spectrum of the inversion in figure 14(b). The energy spectrum of the inversion is in good agreement with the energy spectrum of the slowing-down distribution used to compute the synthetic measurements. Hence inversion assuming isotropy now allows us to measure the $\alpha$-particle energy spectrum down to energies of about $300 \mathrm{keV}$. The same technique also works for the GRS measurements using one spectrometer for energies close to the $2 \mathrm{MeV}$ resonance and higher (figure 14(b)). In particular the fairly sharp kinks in the energy spectrum in the region around the $\alpha$-particle birth energy are reconstructed well using GRS.

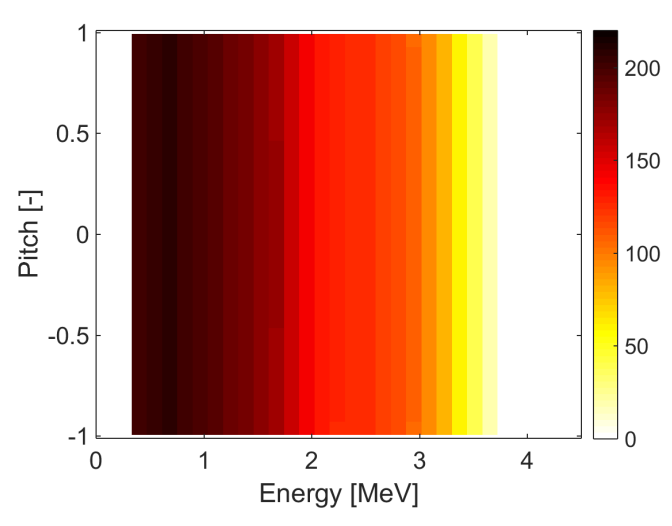

(a)

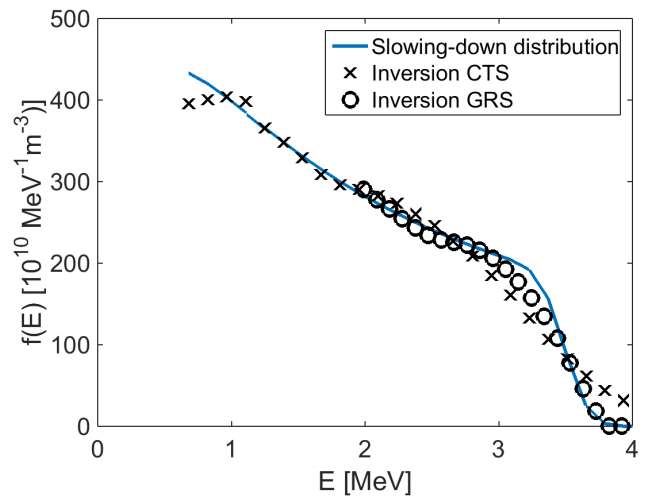

(b)

Figure 14. Measurement of energy spectra by velocity-space tomography based on one measured CTS or GRS spectrum and assuming isotropy. (a) The inversion assuming isotropy penalizes gradients in pitch direction strongly and does therefore not depend on the pitch. (b) The inversion effectively constitutes an $\alpha$-particle energy spectrum which is in excellent agreement with the energy spectrum of the isotropic true solution.

\section{Conclusions}

ITER is going to be equipped with collective Thomson scattering (CTS) and $\gamma$-ray spectrometry (GRS) systems for diagnosing $\alpha$-particles. The velocity space above 1.7 MeV is well diagnosed, but CTS is the only $\alpha$-particle diagnostic with sensitivity below $1.7 \mathrm{MeV}$. Due to the perpendicular viewing direction, the extreme pitches $|p| \gtrsim 0.9-1$ are not observable for $E \lesssim 1.7 \mathrm{MeV}$. CTS is sensitive to $\alpha$-particles down to about $0.3 \mathrm{MeV}$.

GRS observes the plasma completely perpendicularly and CTS almost perpendicularly to the magnetic field. GRS measurements are hence insensitive, and CTS measurements practically insensitive, to the sign of the pitch of the $\alpha$-particles. There are three major groups of fast ions: trapped, co-going and counter-going. Since the sign of 
the pitch cannot be determined with the current set of diagnostics, we cannot tell the groups of co-going and counter-going ions apart. This is a major shortcoming of the fast-ion diagnostic set on ITER, as the physics of fast ions does depend on the sign of the pitch. The $\alpha$-particle velocity distribution function in the plasma center is neoclassically expected to have more co-passing than counter-passing particles for two reasons: The guiding center drifts of co- and counter-going particles are in opposing directions and the birth profile is biased in the direction of the NBI. The resonance condition with Alfvén waves is also different for co- and counter-going particles. Given the importance of understanding the $\alpha$-particle distribution function in ITER, we therefore propose that an additional GRS detector with an oblique viewing angle be installed in ITER.

Velocity-space tomography is feasible for energies above $1.7 \mathrm{MeV}$ where GRS and CTS are sensitive. With the currently planned GRS and CTS diagnostics (lacking an oblique view), only the sign-insensitive distribution function $f(E,|p|)$ can be reconstructed from measurements with realistic noise levels. With an extra oblique GRS view, we could also distinguish the sign of the pitch and infer the full distribution function $f(E, p)$. This would substantially improve our diagnostic capabilities to track any anisotropy in the $\alpha$-particle distribution, for example induced by Alfvén waves.

Below 1.7 MeV velocity-space tomography is not feasible as the GRS is not sensitive so that CTS is practically the only $\alpha$-particle diagnostic. Nevertheless, we demonstrate that the $\alpha$-particle energy spectrum, which is an ITER measurement requirement [77], can be inferred for $E \gtrsim 0.3 \mathrm{MeV}$ based on one CTS spectrum. However, we need to make the approximation that the distribution function is isotropic for measurements of energy spectra based on CTS. The ASCOT code suggests anisotropic $\alpha$-particle distributions, but the deviation from isotropy is at about $10 \%$ acceptable for this measurement.

\section{Acknowledgments}

We thank the ITPA Energetic Particle Physics Topical Group for its support. The work leading to this publication has been funded partially by Fusion for Energy under Specific Grant Agreement F4E-393-SG04. This publication reflects the views only of the authors, and Fusion for Energy cannot be held responsible for any use which may be made of the information contained therein. This work has been partially carried out within the framework of the EUROfusion Consortium and has received funding from the Euratom research and training programme 2014-2018 under grant agreement No 633053. The views and opinions expressed herein do not necessarily reflect those of the ITER Organization, nor the European Commission.

\section{References}

[1] Romanelli F 2015 Nucl. Fusion 55104001

[2] Litaudon X et al 2017 Nucl. Fusion 57102001

[3] Motojima O et al 2015 Nucl. Fusion 55104023 
[4] Shimada M et al Progress in the ITER Physics Basis Chapter 1: Overview and Summary 2007 Nucl. Fusion 47 S1-S17

[5] Giruzzi G et al 2015 Nucl. Fusion 55073002

[6] Wenninger R et al 2015 Nucl. Fusion 55063003

[7] Wenninger R et al 2017 Nucl. Fusion 57016011

[8] Keilhacker M et al 1999 Nucl. Fusion 39 209-234

[9] Heidbrink W and Sadler G 1994 Nucl. Fusion 34 535-615

[10] Wong K L 1999 Plasma Phys. Control. Fusion 41 R1

[11] Jacquinot J et al ITER Physics Basis Chapter 5: Physics of energetic ions 1999 Nuclear Fusion 39 2471-2495

[12] Zweben S et al 2000 Nucl. Fusion 40 91-149

[13] Fasoli A et al Progress in the ITER Physics Basis Chapter 5: Physics of energetic ions 2007 Nucl. Fusion 47 S264-S284

[14] Breizman B N and Sharapov S E 2011 Plasma Phys. Control. Fusion 53054001

[15] Sharapov S et al 2013 Nucl. Fusion 53104022

[16] Gorelenkov N, Pinches S and Toi K 2014 Nuclear Fusion 54125001

[17] Pinches S D et al 2015 Physics of Plasmas 22021807

[18] Chen L and Zonca F 2016 Rev. Mod. Phys. 88015008

[19] McClements K G and Fredrickson E D 2017 Plasma Phys. Control. Fusion 59053001

[20] Yavorskij V et al 2015 J. Fusion Energy 34 774-784

[21] Chugunov I N et al 2011 Nucl. Fusion 51083010

[22] Shevelev A E et al 2013 Nucl. Fusion 53123004

[23] Gin D et al 2014 AIP Conference Proceedings vol 1612 pp 149-152

[24] Nocente et al M 2017 Nucl. Fusion 57076016

[25] Salewski M et al 2008 Rev. Sci. Instrum. 79 10E729

[26] Salewski M et al 2009 Plasma Phys. Control. Fusion 51035006

[27] Salewski M et al 2009 Nucl. Fusion 49025006

[28] Stejner M et al 2012 Nucl. Fusion 52023011

[29] Infante V 2017 Fusion Eng. Des. 123 663-668

[30] Lopes A submitted Fusion Eng. Des. Neutronics analysis of the ITER Collective Thomson Scattering System

[31] Kiptily V G, Cecil F E and Medley S S 2006 Plasma Phys. Control. Fusion 48 R59-R82

[32] Tardocchi M, Nocente M and Gorini G 2013 Plasma Phys. Control. Fusion 55074014

[33] Boyd D A et al 1989 Nucl. Fusion 29 593-604

[34] Jarvis O N et al 1996 Nucl. Fusion 36 1513-1530

[35] Kiptily V G, Cecil F E, Jarvis O N and Mantsinen M J 2002 Nucl. Fusion 42 999-1007

[36] Kiptily V G et al 2003 Rev. Sci. Instrum. 741753

[37] Kiptily V G et al 2004 Physical Review Letters 93115001

[38] Kiptily V G et al 2005 Nucl. Fusion 45 L21-L25

[39] Kiptily V G et al 2009 Nucl. Fusion 49065030

[40] Kiptily V G et al 2012 Plasma Phys. Control. Fusion 54074010

[41] Kiptily V G et al 2013 Plasma and Fusion Research 82502071

[42] Kiptily V G et al 2010 Nucl. Fusion 50084001

[43] Murari A et al 2010 Rev. Sci. Instrum. 81 10E136

[44] Nocente M et al 2010 Rev. Sci. Instrum. 81 10D321

[45] Nocente M et al 2013 IEEE Transactions on Nuclear Science 60 1408-1415

[46] Nocente M et al 2012 Nucl. Fusion 52063009

[47] Tardocchi M et al 2011 Physical Review Letters 107205002

[48] Eriksson J et al 2015 Nucl. Fusion 55123026

[49] Schneider M et al 2016 Nucl. Fusion 56112022

[50] Nocente M et al 2015 Nucl. Fusion 55123009 
[51] Nocente M et al 2012 Nucl. Fusion 52094021

[52] Kiptily V 2015 Nucl. Fusion 55023008

[53] Bindslev H et al 1999 Phys. Rev. Lett. 833206

[54] Salewski M et al 2010 Nucl. Fusion 50035012

[55] Nielsen S K et al 2017 Phys. Scr. 92024001

[56] Rasmussen J et al 2015 Plasma Phys. Control. Fusion 57075014

[57] Rasmussen J et al 2016 Nucl. Fusion 56112014

[58] Nishiura M et al 2014 Nucl. Fusion 54023006

[59] Abramovic I et al 2017 JINST 12 C08015

[60] Abramovic I et al submitted Forward Modelling of Collective Thomson Scattering for Wendelstein 7 - X Plasmas: Electrostatic Approximation

[61] Salewski M et al 2011 Nucl. Fusion 51083014

[62] Salewski M et al 2012 Nucl. Fusion 52103008

[63] Salewski M et al 2013 Nucl. Fusion 53063019

[64] Salewski M et al 2014 Nucl. Fusion 54023005

[65] Geiger B et al 2015 Nucl. Fusion 55083001

[66] Salewski M et al 2015 Plasma Phys. Control. Fusion 57014021

[67] Weiland M et al 2016 Plasma Phys. Control. Fusion 58025012

[68] Jacobsen A S et al 2016 Plasma Phys. Control. Fusion 58045016

[69] Jacobsen A S et al 2016 Plasma Phys. Control. Fusion 58042002

[70] Salewski M et al 2016 Nucl. Fusion 56106024

[71] Jaulmes F et al 2016 Nucl. Fusion 56112012

[72] Salewski M et al 2017 Nucl. Fusion 57056001

[73] Salewski M et al at press Fusion Sci. Tech. 57 Bayesian integrated data analysis of fast-ion measurements by velocity-space tomography

[74] Weiland M et al 2017 Nucl. Fusion 57116058

[75] Geiger B et al 2017 Plasma Phys. Control. Fusion 59115002

[76] Moseev D et al submitted Fast-ion diagnostics in magnetically confined plasmas

[77] Donné A J H et al Progress in the ITER Physics Basis Chapter 7: Diagnostics 2007 Nucl. Fusion 47 S337-S384

[78] Stagner L and Heidbrink W 2017 Phys. Plasmas 24092505

[79] Kiptilyj V G 1990 Fusion Science and Technology 18 583-590

[80] Cazzaniga C et al 2015 Nucl. Instrum. Methods A 778 20-25

[81] Nocente M et al 2016 Rev. Sci. Instrum. 87 11E714

[82] Heidbrink W W et al 2007 Plasma Phys. Control. Fusion 49 1457-1475

[83] Jacobsen A S et al 2015 Nucl. Fusion 55053013

[84] Salewski M et al 2015 Nucl. Fusion 55093029

[85] Salewski M et al 2016 Nucl. Fusion 56046009

[86] Jacobsen A S et al 2017 Rev. Sci. Instrum. 88073506

[87] Gaffey J D 1976 J. Plasma Phys. 16 149-169

[88] Pinches S D et al 2004 Plasma Phys. Control. Fusion 46 B187-B200

[89] Hansen P C 2010 Discrete Inverse Problems (SIAM, Philadelphia, US)

[90] Hirvijoki E et al 2014 Comp. Phys. Comm. 185 1310-1321

[91] Kurki-Suonio T et al 2016 Nucl. Fusion 56112024

[92] Varje J et al 2016 Nucl. Fusion 56046014

[93] Porcelli F et al 1994 Phys. Plasmas 1470

[94] Heidbrink W W et al 2008 Nucl. Fusion 48084001 